\title{
Simultaneous Spectral Energy Distribution and Near-infrared Interferometry Modeling of HD 142666
}

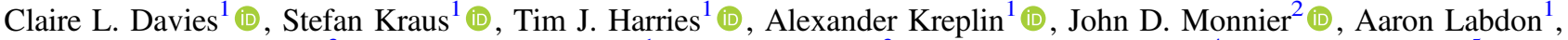 \\ Brian Kloppenborg $^{3}$ (i), David M. Acreman ${ }^{1}$ (i) , Fabien Baron $^{3}$ (i), Rafael Millan-Gabet ${ }^{4}$ (i), Judit Sturmann ${ }^{5}$, \\ Laszlo Sturmann ${ }^{5}$, and Theo A. Ten Brummelaar ${ }^{5}$ \\ ${ }^{1}$ Astrophysics Group, School of Physics, University of Exeter, Stocker Road, Exeter, EX4 4QL, UK; cdavies@astro.ex.ac.uk \\ ${ }^{2}$ Department of Astronomy, University of Michigan, Ann Arbor, MI 48109, USA \\ ${ }^{3}$ Department of Physics and Astronomy, Georgia State University, Atlanta, GA, USA \\ ${ }^{4}$ Infrared Processing and Analysis Center, California Institute of Technology, Pasadena, CA 91125, USA \\ ${ }^{5}$ The CHARA Array of Georgia State University, Mount Wilson Observatory, Mount Wilson, CA 91203, USA \\ Received 2018 August 10; accepted 2018 August 28; published 2018 October 5
}

\begin{abstract}
We present comprehensive models of the Herbig Ae star, HD 142666, which aim to simultaneously explain its spectral energy distribution (SED) and near-infrared (NIR) interferometry. Our new submilliarcsecond resolution CHARA (CLASSIC and CLIMB) interferometric observations, supplemented with archival shorter baseline data from VLTI/PIONIER and the Keck Interferometer, are modeled using centrosymmetric geometric models and an axisymmetric radiative transfer code. CHARA's $330 \mathrm{~m}$ baselines enable us to place strong constraints on the viewing geometry, revealing a disk inclined at $58^{\circ}$ from face-on with a $160^{\circ}$ major axis position angle. Disk models imposing vertical hydrostatic equilibrium provide poor fits to the SED. Models accounting for disk scale height inflation, possibly induced by turbulence associated with magnetorotational instabilities, and invoking grain growth to $\gtrsim 1 \mu \mathrm{m}$ size in the disk rim are required to simultaneously reproduce the SED and measured visibility profile. However, visibility residuals for our best model fits to the SED indicate the presence of unexplained NIR emission, particularly along the apparent disk minor axis, while closure phase residuals indicate a more centrosymmetric emitting region. In addition, our inferred $58^{\circ}$ disk inclination is inconsistent with a disk-based origin for the UX Ori-type variability exhibited by HD 142666. Additional complexity, unaccounted for in our models, is clearly present in the NIR-emitting region. We propose that the disk is likely inclined toward a more edge-on orientation and/or an optically thick outflow component also contributes to the NIR circumstellar flux.
\end{abstract}

Key words: infrared: stars - protoplanetary disks - stars: formation - stars: individual (HD 142666 ) - stars: variables: T Tauri, Herbig Ae/Be - techniques: interferometric

\section{Introduction}

Circumstellar disks are ubiquitous across all masses of star formation (e.g., Andrews et al. 2013; Ricci et al. 2014; Ilee et al. 2016; Kraus et al. 2017; Lazareff et al. 2017), a consequence of the conservation of angular momentum during gravitational collapse. These disks provide the building materials and the natal environment for planets to form and evolve in. The reprocessing of starlight by dust in the innermost regions of protoplanetary disks produces strong near-infrared (NIR) continuum emission, in excess of that expected from a stellar photosphere. Developments in the field of NIR interferometry during the late 1990s enabled the first spatially resolved observations of the circumstellar structure of Herbig $\mathrm{Ae} / \mathrm{Be}$ stars - the precursors to intermediate-mass stars (Herbig 1960; Strom et al. 1972) - to be obtained. The milliarcsecond (mas) resolution offered by the Infrared Optical Telescope Array (IOTA) and Palomar Testbed Interferometer (PTI) showed that the inner disk regions did not extend down to the stellar surface (e.g., Millan-Gabet et al. 1999; Akeson et al. 2000), in agreement with prior spectral energy distribution (SED) modeling (Hillenbrand et al. 1992). As the number of Herbig Ae/Be stars observed with NIR interferometry increased, a relationship between the host star luminosity and the characteristic size of the NIR-emitting region emerged (Monnier \& Millan-Gabet 2002). The slope of this sizeluminosity relationship suggests that the NIR-emitting region arises from a dust sublimation rim at a temperature of $\sim 1800 \mathrm{~K}$ (Lazareff et al. 2017).

Early disk models incorporating a dust sublimation rim used a vertical-wall approximation (Dullemond et al. 2001; Natta et al. 2001). However, the strong viewing angle dependency of the NIR emission associated with such a model is in conflict with the similar levels of NIR excess observed among Herbig $\mathrm{Ae} / \mathrm{Be}$ stars over a wide range of disk inclination angles (Natta et al. 2001; Dominik et al. 2003). In addition, the significant closure phase $\left(\phi_{\mathrm{CP}}\right)$ signals associated with the strongly asymmetric NIR brightness distribution in vertical rim models was not observed (Monnier et al. 2006; Kraus et al. 2009). Instead, the curvature of the inner rim is understood to arise due to the dependence of the dust sublimation temperature and grain cooling efficiency on, for example, the gas density, the size distribution of dust grains, grain-growth-induced vertical settling, and the relative abundance of different grain compositions (Isella \& Natta 2005; Tannirkulam et al. 2007; Kama et al. 2009; McClure et al. 2013).

The picture was further complicated with the first sub-mas NIR observations of Herbig Ae/Be stars, made possible with the $\sim 330 \mathrm{~m}$ baselines of the Center for High Angular Resolution Astronomy (CHARA) Array. Through their observations of MWC 275 and AB Aur, Tannirkulam et al. (2008b) found that the "bounce" in the secondary visibility lobe predicted by curved rim models was not observed. Instead, to explain the relatively flat profiles of the observed second 
visibility lobes, an additional NIR-emitting component interior to the silicate dust sublimation front was required. Further evidence for this has been reported in studies using NIR spectro-interferometry (e.g., Kraus et al. 2008; Eisner et al. 2009), high-resolution spectroscopy (e.g., Ilee et al. 2014), and photometry (e.g., Fischer et al. 2011). The nature of this material remains unclear with plausible suggestions including a hot gas reservoir and/or more refractory grain species (Tannirkulam et al. 2008a; Eisner et al. 2009).

Here, we focus on the shape, location, and viewing geometry of the circumstellar disk of the Herbig Ae star, HD 142666 (spectral type A8Ve; Meeus et al. 1998). The IR excess of HD 142666 (common aliases include V1026 Sco), first identified by Walker \& Wolstencroft (1988), has previously been studied using NIR and mid-IR (MIR) interferometers with operational baselines $\lesssim 100 \mathrm{~m}$. The characteristic size of the $H$ - and $K$-band-emitting regions observed with the Keck Interferometer (KI), VLTI/AMBER, and VLTI/PIONIER (henceforth referred to as AMBER and PIONIER, respectively) is consistent with that expected from dust sublimation $(\sim 0.4 \mathrm{au}$ at a stellar distance of $150 \mathrm{pc}$; Monnier et al. 2005; Lazareff et al. 2017) while the MIR emission observed with VLTI/ MIDI is more extended than predicted by typically adopted temperature gradient models, suggesting a narrow, dust-free gap is present within the inner few astronomical units of the disk (Schegerer et al. 2013; Vural et al. 2014). However, the usual features indicative of optically thin disk regions or disk cavities are not seen in the SED of HD 142666 (Dominik et al. 2003), meaning the disk is not typically considered to be (pre-)transitional. Intermediate disk inclinations for HD 142666 have been indicated via NIR and MIR interferometry $\left(48^{\circ} .6_{-3.6}^{+2.9}\right.$, Vural et al. 2014; $\sim 60^{\circ}$, Lazareff et al. 2017), SED analysis ( $\sim 55^{\circ}$, Dominik et al. 2003$)$, and ALMA $\left(\sim 60^{\circ}\right.$, Rubinstein et al. 2018). VLT/NACO differential imaging and ALMA indicate that the disk major axis position angle ${ }^{6}, \mathrm{PA}_{\text {major }}$, is oriented along a nearly north-south direction $\left(\sim 180^{\circ}\right.$, Garufi et al. 2017; $161^{\circ}$, Rubinstein et al. 2018)).

We present new, high-resolution NIR interferometric data of HD 142666 obtained using the CLASSIC two-telescope and CLIMB three-telescope beam combiners of the CHARA Array (ten Brummelaar et al. 2013). Section 2 details our CHARA observations and the supplementary, shorter baseline NIR interferometry retrieved from the archives. With its $\sim 331 \mathrm{~m}$ maximum baseline length, our CHARA observations offer us the opportunity to distinguish between different curved rim models to understand the dominant process of rim curvature in the disk of HD 142666. Our analysis builds upon that of Tannirkulam et al. (2008a), who used the TORUS Monte Carlo radiative transfer code (Harries 2000) to model the NIR interferometric visibilities of two other Herbig Ae stars-MWC 275 and $\mathrm{AB}$ Aur (spectral types of $\mathrm{A} 1$ and $\mathrm{A} 0$, respectively; Mora et al. 2001; Hernández et al. 2004)—obtained with CHARA/CLASSIC (henceforth referred to as CLASSIC). In addition to considering a later type Herbig Ae star, (i) our $(u, v)$ plane coverage is much improved compared to the Tannirkulam et al. (2008a) study, (ii) we probe $H$ - as well as $K$-band emission, and (iii) with the addition of CHARA/CLIMB (henceforth referred to as CLIMB) data, we use $\phi_{\mathrm{CP}}$ information to further constrain our modeling.

\footnotetext{
6 Quoted disk position angles, $\mathrm{PA}_{\text {major }}$, are for the disk major axis, measured east of north.
}

A twofold approach is used in our analysis. First, we constrain the stellar flux contribution to the NIR flux by fitting stellar atmosphere models to optical photometry and employ centrosymmetric geometric models to constrain the viewing geometry of the NIR-emitting region. We then build on these models using the TORUS Monte Carlo radiative transfer code to explore the physical bases behind the location and shape of the observed inner disk rim. Our modeling approach is outlined in Section 3, while the results of our geometric and radiative transfer analysis are presented in Sections 4 and 5, respectively. In Section 6, we discuss our results in the context of grain growth to micron sizes in the inner rim and comment on indirect evidence for further complexity in the NIR-emitting region.

\section{Observations and Complementary Archival Data}

\subsection{CHARA Interferometry}

CHARA is a Y-shaped array of six $1 \mathrm{~m}$ class telescopes located at Mount Wilson Observatory offering operational baselines between 34 and $331 \mathrm{~m}$ (ten Brummelaar et al. 2005). CLASSIC and CLIMB (ten Brummelaar et al. 2013) were used to obtain $K$-band observations of HD 142666 between 2009 June and 2013 June. Additional $H$-band observations of HD 142666 were obtained with CLIMB in 2014 May and June.

A variety of telescope configurations were used during the observing campaign with a maximum projected baseline length of $313 \mathrm{~m}$ (corresponding to an angular resolution ${ }^{7}$ of 0.70 mas). Further details regarding the individual observations are provided in Table 1 . The resulting $(u, v)$ plane coverage is displayed in Figure 1.

The CLASSIC and CLIMB data were reduced using pipelines developed at the University of Michigan which are better suited to recovering faint fringes for low visibility data than the standard CHARA reduction pipeline of ten Brummelaar et al. (2012). The waterfall plot of raw data scans was first inspected for instrumental or observational effects such as drifting scans or flux dropout on one or more telescope, for example. Any scans displaying these effects were flagged and rejected. In the majority of cases, this affected at most 5\%$10 \%$ of scans. Extra care was taken on the few occasions where drift or low signal-to-noise ratio dominated the majority of scans. In these cases, the affected scans were carefully flagged while the power spectrum, averaged over the retained scans, was inspected for a signal. After this process, the foreground, background, and flux recorded for each baseline pair were each inspected for flux dropout. Finally, the power spectrum for each telescope pair and CLIMB output (P0, P1, and P2; see ten Brummelaar et al. 2012) was inspected and the background level set manually. This results in one $\phi_{\mathrm{CP}}$ for each baseline triplet, three estimates of the square visibility, $V^{2}$, for one baseline pair and two estimates of $V^{2}$ for the remaining two CLIMB baseline pairs.

Calibration of the $V^{2}$ and $\phi_{\mathrm{CP}}$ measurements was made using standard stars observed before and/or after each science observation. None of the calibrators used are known binary systems. As a further check, the $\phi_{\mathrm{CP}}$ signals of each of the calibrators observed more than once were inspected: no signatures of binarity were found. The uniform diameters (UDs) of each calibrator, obtained from JMMC SearchCal

\footnotetext{
$7 \lambda / 2 B$, with $\lambda$ the operational wavelength and $B$ the projected baseline length.
} 
Table 1

CHARA Observation Log

\begin{tabular}{lcccl}
\hline \hline Date (UT) & Beam Combiner & Stations & Filter & Calibrator(s) \\
\hline 2009 Jun 24 & CLASSIC & S2 E2 & $K$ & 1,2 \\
2010 Jun 15 & CLASSIC & S2 W1 & $K$ & 3,4 \\
2011 Jun 15 & CLIMB & E1 W1 W2 & $K$ & 3 \\
2011 Jun 20 & CLIMB & (E1) ${ }^{\mathrm{a}} \mathrm{W} 1 \mathrm{~W} 2$ & $K$ & 3 \\
2011 Jun 23 & CLIMB & S1 W1 W2 & $K$ & 3,5 \\
2011 Jun 25 & CLIMB & S1 E2 W2 & $K$ & 6,7 \\
2011 Jun 27 & CLIMB & S1 W1 W2 & $K$ & 6 \\
2011 Jun 28 & CLIMB & S1 E2 W2 & $K$ & 6 \\
2011 Aug 03 & CLIMB & S1 E2 W1 & $K$ & 3,5 \\
2012 Jun 29 & CLIMB & E1 E2 W1 & $K$ & 3 \\
2012 Jul 01 & CLIMB & S1 S2 W1 & $K$ & 6 \\
2013 Jun 10 & CLIMB & S1 W1 W2 & $K$ & 8 \\
2013 Jun 13 & CLIMB & S1 W1 W2 & $K$ & 9,10 \\
2013 Jun 14 & CLIMB & S2 E2 W2 & $K$ & 8,9 \\
2013 Jun 16 & CLIMB & E2 W1 W2 & $K$ & 11 \\
2014 May 28 & CLIMB & S2 E2 W2 & $H$ & 3 \\
2014 May 29 & CLIMB & S2 E2 W2 & $H$ & 3,5 \\
2014 May 30 & CLIMB & S2 E2 W2 & $H$ & 3,5 \\
2014 Jun 04 & CLIMB & S2 E2 W1 & $H$ & 3,5 \\
2014 Jun 09 & CLIMB & E1 E2 W1 & $H$ & 5 \\
2014 Jun 10 & CLIMB & S2 W1 W2 & $H$ & 3,5 \\
\hline
\end{tabular}

Notes. Calibrators and their UD diameters: (1) HD 141465, $0.28 \pm 0.02$ mas; (2) HD 143766, $0.311 \pm 0.022$ mas; (3) HD 140990, $0.230 \pm 0.016$ mas;

(4) HD 141597, $0.24 \pm 0.05$ mas; (5) HD 143616, $0.222 \pm 0.016$ mas; (6) HD 148211, $0.250 \pm 0.018$ mas; (7) HD 152429, $0.272 \pm 0.019$ mas; (8) HD 148198, $0.255 \pm 0.018$ mas; (9) HD 144766, $0.324 \pm 0.023$ mas; (10) HD 145809, $0.417 \pm 0.029$ mas; (11) HD 141937, $0.307 \pm 0.022$ mas.

${ }^{\text {a }}$ CLIMB operating as a two-telescope beam combiner.

(Bonneau et al. 2006, 2011), where available, or getCal, ${ }^{8}$ are listed in Table 1. The transfer function across the full observation sequence was inspected to ensure its flatness. Finally, the multiple estimates of the calibrated $V^{2}$ on each baseline pair were checked for consistency before a weightedaverage value was computed. For our analysis, we thus have one estimate of $V^{2}$ for each baseline pair. The raw and calibrated data will be made available in oifits format (Pauls et al. 2005; Duvert et al. 2017) through the CHARA archive (J. Jones et al. 2018, in preparation) and the Optical interferometry Database (OiDb; Haubois et al. 2014) of the JMMC following publication.

\subsection{Supplementary Archival Interferometry}

To better constrain the geometry of the disk, we supplemented our long-baseline CLASSIC and CLIMB data with shorter baseline archival NIR interferometry. Calibrated PIONIER (Le Bouquin et al. 2011) data for HD 142666 , originally published in Lazareff et al. (2017; program IDs 190. C-0963, 088.D-0185, and 088.C-0763), were retrieved from the OiDb. PIONIER data from UT date 2013 June 17, not available on the $\mathrm{OiDb}$, were also provided by $\mathrm{B}$. Lazareff (2018, private communication). KI (Colavita et al. 2013) data were retrieved from the Keck Observatory Archive. The wideband KI data were calibrated using the NExScI Wide-band Interferometric Visibility Calibration (wbCalib v1.4.4) tool with the flux bias correction and ratio correction options selected. Table 2 provides further details on the collated data

\footnotetext{
http://nexsci.caltech.edu/software/getCal/
}
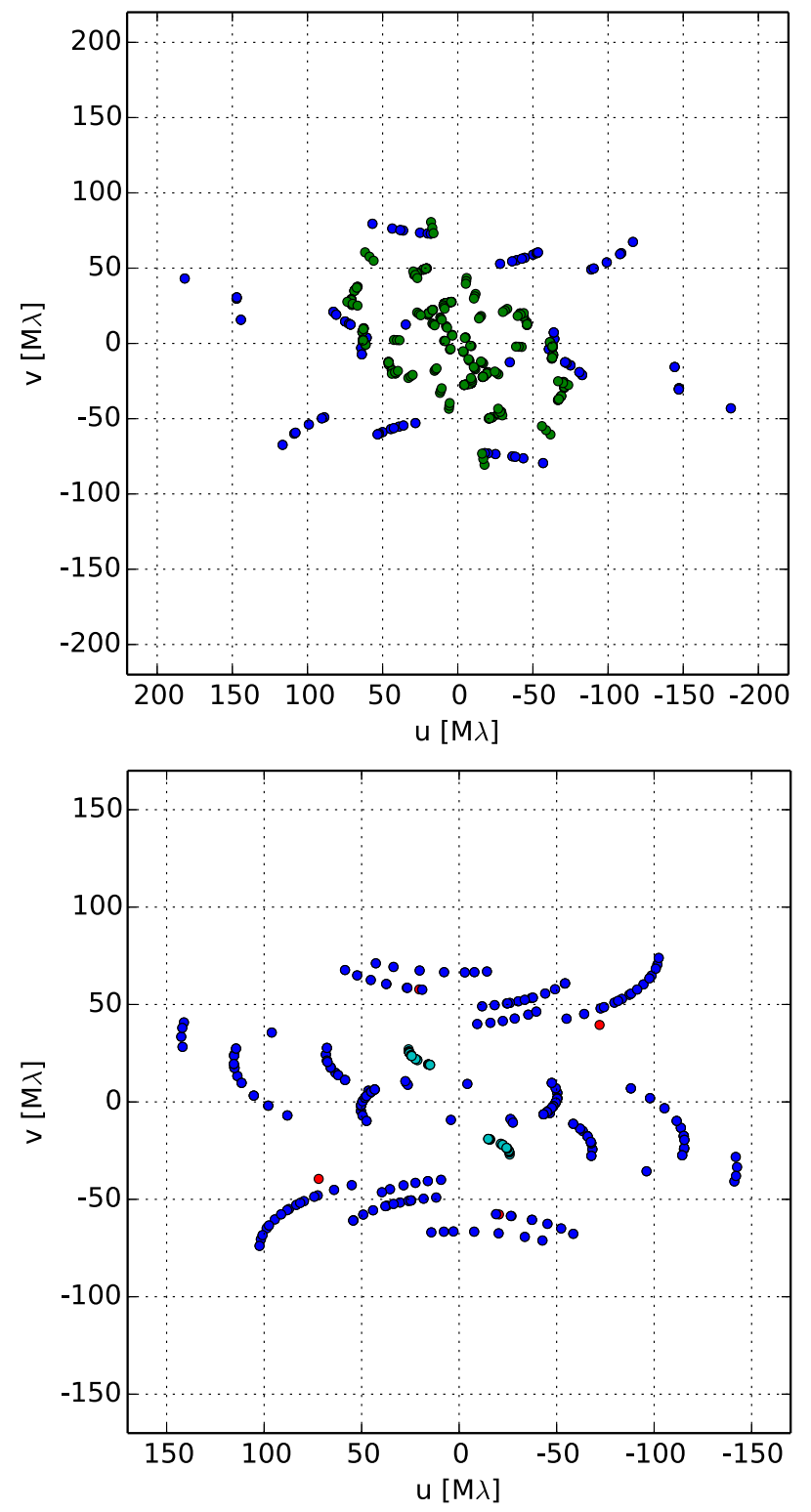

Figure 1. $(u, v)$ plane coverage for HD 142666 in the $H$ - (top) and $K$-bands (bottom). Positive values of $v$ and $u$ correspond to north and east, respectively. Our new CLASSIC and CLIMB data (see Table 1) are shown as red and blue points, respectively. Supplementary archival NIR interferometric data, listed in Table 2, are also indicated: KI (cyan); PIONIER (green).

and, for the occasions where the data required (re-)reduction, also the names and UD diameters of the standard stars used to calibrate $V^{2}$ and $\phi_{\mathrm{CP}}$.

\section{Modeling Methodology}

We model the location and extent of the circumstellar NIRemitting region of HD 142666 using the Monte Carlo radiative transfer code, TORUS (Harries 2000; Harries et al. 2004; Kurosawa et al. 2006; Tannirkulam et al. 2007). Exploring viewing geometries using TORUS would be computationally expensive, so, to allow for more rapid exploration, we employed a series of geometric models to determine the bestfit inclinations and position angles. In the subsections that follow, we outline the methodology adopted in both our analyses. 
Table 2

Supplementary Archival NIR Interferometric Data

\begin{tabular}{|c|c|c|c|c|c|}
\hline Instrument & Observation Date (UT) & Program ID & Stations & Filter & Calibrator(s) \\
\hline \multirow{3}{*}{ VLTI PIONIER } & 2012 Mar 29 & 088.C-0763 & A1 G1 I1 K0 & $H$ & $\cdots$ \\
\hline & 2013 Jun 06 & 190.C-0963 & A1 G1 J3 K0 & $H$ & $\ldots$ \\
\hline & 2013 Jul 03 & 190.C-0963 & A1 B2 C1 D0 & $H$ & $\cdots$ \\
\hline \multirow[t]{3}{*}{ KI V2-SPR } & 2004 Mar 05 & 13 & $\mathrm{~K} 1 \mathrm{~K} 2$ & $K$ & 1 \\
\hline & 2007 Jul 02 & 32 & $\mathrm{~K} 1 \mathrm{~K} 2$ & $K$ & 2,3 \\
\hline & 2012 May 03 & 57 & $\mathrm{~K} 1 \mathrm{~K} 2$ & $K$ & 7 \\
\hline
\end{tabular}

Note. Calibrators listed in column 7 for the instances where a re-reduction of the data was required: (1) HD 134967, $0.15 \pm 0.01$ mas; (2) HD 139364, $0.323 \pm 0.023$ mas; (3) HD 141465, $0.28 \pm 0.02$ mas; (4) HD 142301, $0.159 \pm 0.011$ mas; (5) HD $1437660.311 \pm 0.022$ mas; (6) HD $145809,0.417 \pm$ 0.029 mas; (7) HD 141597, $0.24 \pm 0.05$ mas.

\subsection{Geometric Modeling of the Visibilities}

The visibility of the circumstellar emission is

$$
V_{\mathrm{CS}}=\frac{\left|V_{\mathrm{obs}}\left(F_{\star}+F_{\mathrm{CS}}\right)-V_{\star} F_{\star}\right|}{F_{\mathrm{CS}}}
$$

where $V_{\mathrm{obs}}$ is the observed visibility, and $F_{\star}$ and $F_{\mathrm{CS}}$ refer to the stellar and circumstellar flux contributions, respectively. The stellar emission component of HD 142666 is expected to be unresolved as the stellar radius is much smaller than the length scales we are able to probe. As such, we set the visibility of the stellar component to unity, i.e., $V_{\star}=1$.

We required an independent assessment of $F_{\star}$ at the $H$ - and $K$-bands to avoid degeneracies associated with fitting both the characteristic size of the emitting region and $F_{\star}$ simultaneously (see, for example, Lazareff et al. 2017). Multiwavelength photometry was retrieved from the literature while a postprocessed, flux-calibrated Spitzer Infrared Spectrograph (IRS; Houck et al. 2004) spectrum (Keller et al. 2008; AORkey 3586816) was retrieved from the Spitzer Heritage Archive. The full list of collated photometry, together with the individual references, is presented in Table 9 in the Appendix. The photospheric portion (Johnson- $B,-V$, and Cousins- $I_{C}$ wavebands) of the SED constructed for HD 142666 was then fit using Kurucz (1979) model atmospheres appropriate for the star (see Section 4).

The measured $\phi_{\mathrm{CP}}$ were inspected for deviations from centrosymmetry. While no significant indication for non-zero $\phi_{\mathrm{CP}}$ was visible in the full $H$-band data set (CLIMB +PIONIER), a possible deviation from centrosymmetry is suggested by the full $K$-band data set (CLIMB). Assuming the NIR emission from HD 142666 emanates from the inner regions of an inclined disk, a non-zero $\phi_{\mathrm{CP}}$ may indicate a degree of skewness in the disk emission caused by selfshielding, for example. Alternative scenarios include, but are not restricted to, the presence of regions with enhanced brightness, possibly indicating an increased disk scale height (i.e., disk warp) or alluding to the presence of additional companions. If these features co-orbit with the disk, their dynamical timescales may be smaller than the four-year timescale over which the $\phi_{\mathrm{CP}}$ were obtained.

In Figure 2, we plot the observed $\phi_{\mathrm{CP}}$ against the maximum spatial frequency probed by each triplet of baseline vectors, split by observational epoch and waveband. In each panel, the reduced- $\chi^{2}\left(\chi_{\mathrm{r}}^{2}\right)$ value computed for a centrosymmetric model

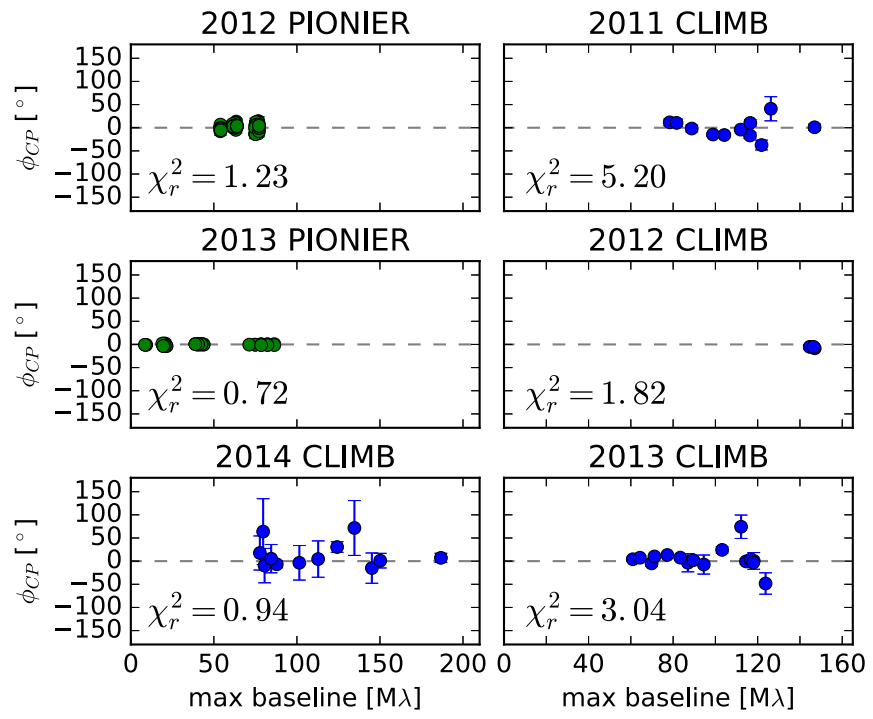

Figure 2. Closure phase as a function of the maximum spatial frequency probed by each closed triangle of baseline vectors. Left-hand panels contain the $H$-band PIONIER and CLIMB data while right-hand panels contain the $K$-band CLIMB data, each split by the year of observation (see Tables 1 and 2). Data points are colored as in Figure 1. The $\chi_{\mathrm{r}}^{2}$ for centrosymmetric models $\left(\phi_{\mathrm{CP}}=0^{\circ}\right.$ on all spatial scales) is displayed in the lower left corner of each panel.

( $\phi_{\mathrm{CP}}=0$ at all spatial frequencies) is displayed in the top lefthand corner. Although there may be an indication for deviation from centrosymmetry in the 2011 and $2013 \mathrm{~K}$-band data, the $\phi_{\mathrm{CP}}=0^{\circ}$ model provides a good fit to all epochs. Thus, to estimate the geometry of the $H$ - and $K$-band-emitting regions, we restrict our analysis to centrosymmetric models.

In Section 4, we consider two geometric models for the brightness distribution. Both of these use a point-source component to model the stellar flux contribution and assume, for simplicity, that all non-stellar NIR emission arises from the innermost regions of a disk. In the first model, a thin ring of emission is used to emulate the disk component, corresponding to the emission expected from a centrally illuminated vertical wall. The free parameters of this point-source-plus-ring $(\mathrm{PS}+\mathrm{R})$ model are the ring radius, $R$; its inclination, $i$ (where $0^{\circ}$ corresponds to a face-on viewing geometry); and its major axis position angle, $\mathrm{PA}_{\text {major }}$ (measured east of north). In the second model, the disk emission is approximated as a Gaussiansmoothed ring, avoiding the sharp edges of the ring model and 
Table 3

TORUS Radiative Transfer Models Summary Showing Grain Fractions by Mass, Dust Scale Height $\left(h_{0, \text { dust }}\right)$ Relative to Gas Scale Height $\left(h_{0 \text {,gas }}\right)$, and Sublimation Temperature $\left(T_{\text {sub }}\right)$

\begin{tabular}{lccc}
\hline \hline & S:small & S:large & THM07 \\
\hline $0.1 \mu$ m grain fraction $(\%)$ & 100 & 0 & 90 \\
$1.2 \mu$ m grain fraction $(\%)$ & 0 & 100 & 10 \\
$0.1 \mu$ m grain $h_{0, \text { dust }}\left(h_{0, \text { gas }}\right)$ & 1.0 & $\ldots$ & 1.0 \\
$1.2 \mu$ m grain $h_{0, \text { dust }}\left(h_{0, \text { gas }}\right)$ & $\ldots$ & 1.0 & 0.6 \\
$T_{\text {sub }}(\mathrm{K})$ & $G \rho^{\gamma}(r, z)$ & $G \rho^{\gamma}(r, z)$ & 1400 \\
\hline
\end{tabular}

corresponding to a more spatially extended NIR-emitting region. The FWHM of the Gaussian used in the convolution remains a free parameter in the fitting procedure. These pointsource-plus-smoothed ring models are henceforth referred to as $\mathrm{PS}+\mathrm{SR}$.

During the fitting procedure, errors on the best-fit parameters (found via $\chi^{2}$ minimization) were estimated via bootstrapping. A thousand new realizations of the original visibility data sets were created and fed through the same modeling procedure as the original data. The initial values of the parameters in the fitting remained consistent between data sets in the same waveband and throughout the bootstrapping process. Histograms were created from the resulting bootstrapped model outputs, and errors were estimated from $1 \sigma$ Gaussian fits to each histogram.

\subsection{Monte Carlo Radiative Transfer Modeling with TORUS}

The TORUS Monte Carlo radiative transfer code uses the Lucy (1999) algorithm to compute radiative equilibrium on a two-dimensional, cylindrical adaptive mesh grid. Assuming that all of the circumstellar NIR emission of HD 142666 arises from a disk, we prescribe the TORUS models as follows. The initial density structure of the gas component of the disk, $\rho(r, z)$, is based on the $\alpha$-disk prescription of Shakura \& Sunyaev (1973):

$$
\rho(r, z)=\frac{\Sigma(r)}{h(r) \sqrt{2 \pi}} \exp \left\{-\frac{1}{2}\left[\frac{z}{h(r)}\right]^{2}\right\} .
$$

Here, $r$ and $z$ are the radial distance into the disk and the vertical height above the disk midplane, respectively. The parameters $h(r)$ and $\Sigma(r)$ describe the scale height,

$$
h(r)=h_{0, \mathrm{gas}}\left(\frac{r}{100 \mathrm{au}}\right)^{\beta},
$$

and the surface density,

$$
\Sigma(r)=\Sigma_{0, \mathrm{gas}}\left(\frac{r}{100 \mathrm{au}}\right)^{-p},
$$

of the gas component of the disk, respectively. The constants $h_{0, \text { gas }}$ and $\Sigma_{0, \text { gas }}$ are each equated at $r=100 \mathrm{au}$. We keep $p=1.0$ fixed in all models.

The disk is passively heated by a single star located at the grid center and is assumed to be in local thermodynamic equilibrium. The temperature structure of the disk and the location and shape of the dust sublimation region are established in an iterative manner using the Lucy (1999) algorithm. To investigate the shape of the inner rim of the disk, we use two different parameterizations of the sublimation region facilitated by TORUS. These are summarized in Table 3 and discussed briefly in Section 3.2.1, and the interested reader is referred to Tannirkulam et al. (2007) for further details. In both cases, an $e$-folding factor of $10 \mathrm{~K}$ to the dust sublimation temperature has been introduced to enable convergence.

TORUS solves for radiative equilibrium with or without imposing vertical hydrostatic equilibrium. If vertical hydrostatic equilibrium is imposed, the vertical structure of the disk is modified via the equation of vertical hydrostatic equilibrium according to an adapted form of the Walker et al. (2004) algorithm following each Lucy (1999) iteration (Tannirkulam et al. 2007). The process of establishing a converged temperature and dust sublimation structure is then repeated. Typically, these models converge after the third iteration of imposing vertical hydrostatic equilibrium.

Following convergence, a separate Monte Carlo algorithm is used to compute model SEDs and $H$ - and $K$-band images based on the optical properties of the dust species used in the particular model (Harries 2000; see Section 3.2.1 for details of the grain prescriptions used in our models). All model outputs were computed at a distance of 150 pc (Lindegren et al. 2016) based on the distance inferred from the Gaia DR1 parallax and consistent with that inferred from the more recent Gaia DR2 parallax (148 $\pm 1 \mathrm{pc}$; Gaia Collaboration et al. 2016, 2018; Bailer-Jones et al. 2018), and at the best-fit disk inclinations found through our geometric modeling (see Section 4).

Visibility amplitudes and phases were extracted from the model images at $\mathrm{PA}_{\text {base }}$ and baseline lengths corresponding to the $(u, v)$ plane positions of our interferometric data (see Figure 1). Model $\phi_{\mathrm{CP}}$ were then computed from the sum of the visibility Fourier phases over each closed triangle of baseline vectors. Due to the combined effects of the model image resolution (which introduces errors when the image is rotated and via the interpolation between pixels to the correct baseline length) and numerical estimation of the complex visibilities, our procedure for estimating model $\phi_{\mathrm{CP}}$ introduces an uncertainty of $\sim 1^{\circ}$. This is within our CLIMB measurement uncertainties.

\subsubsection{Dust Grain Prescription and Implementation of Rim Curvature}

The location of the disk inner rim is controlled by the dust species with the highest sublimation temperature, $T_{\text {sub }}$, and greatest cooling efficiency (Isella \& Natta 2005; Kama et al. 2009). We limit our analysis to astronomical silicate grains, which sublimate at temperatures consistent with those inferred from the NIR size-luminosity relation (Pollack et al. 1994). The grains are modeled as homogeneous spheres with a mass density of $3.3 \mathrm{~g} \mathrm{~cm}^{-3}$ (Kim et al. 1994) and optical constants prescribed by Draine (2003), which differ from those of Draine \& Lee (1984) only in the details.

In one set of TORUS models, an inner disk rim curvature arises due to the dependence of $T_{\text {sub }}$ on the local gas density (Pollack et al. 1994; Isella \& Natta 2005):

$$
T_{\text {sub }}=G \rho^{\gamma}(r, z)
$$

Here, $\gamma=1.95 \times 10^{-2}$ and the constant $G=2000 \mathrm{~K}$ for silicate grains. Grains larger than $\sim 1.3 \mu \mathrm{m}$ in size do not contribute sufficiently to the disk opacity and thus do not play a role in determining the location of the dust rim (Isella \& Natta 2005). As such, we adopt two different grain size prescriptions for these models: one set with small grains $(0.1 \mu \mathrm{m}$ in size $)$ and the other with large grains $(1.2 \mu \mathrm{m}$ in size $)$, 
Table 4

Adopted Stellar Parameters

\begin{tabular}{|c|c|c|c|c|c|c|c|c|c|c|c|}
\hline SpT & $T_{\text {eff }}(\mathrm{K})$ & $\log g$ & $\log Z$ & $d(\mathrm{pc})$ & $L_{\star} / L_{\odot}$ & $A_{V}$ & $R_{\star} / R_{\odot}$ & $M_{\star} / M_{\odot}$ & $F_{\star, \mathrm{H}}$ & $F_{\star, \mathrm{K}}$ & $R_{\text {out }}(\mathrm{au})$ \\
\hline A8 & 7500 & 4.3 & 0.2 & 150 & 19.3 & 1.63 & 2.42 & 1.97 & 0.61 & 0.35 & 60 \\
\hline
\end{tabular}

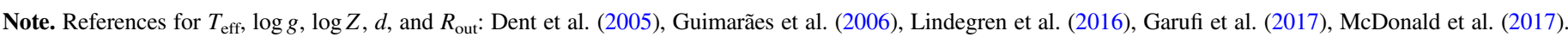

consistent with the original Isella \& Natta (2005) study. As these models use a single grain size, they are henceforth referred to as the S:small and S:large models, respectively.

In our other set of TORUS models, rim curvature arises due to the dependence of dust sublimation on the grain-sizedependent cooling efficiency (Kamp \& Dullemond 2004; Kama et al. 2009) and settling, as originally prescribed in Tannirkulam et al. (2007). These are henceforth referred to as THM07 models. We adopt a thermally coupled mixture of 0.1 and $1.2 \mu \mathrm{m}$ grains in a ratio of 9:1 by mass in favor of the small grains. $\quad T_{\text {sub }}=1400 \mathrm{~K}$ is used for both grain sizes for consistency with the original Tannirkulam et al. (2008a) study of the Herbig Ae stars AB Aur and MWC 275. We also limit the disk scale height of the $1.2 \mu \mathrm{m}$ grains to $60 \%$ that of the gas, $h_{0, \text { gas }}$, while the $0.1 \mu \mathrm{m}$ grains inhabit the full $h_{0 \text {,gas }}$ range.

\subsubsection{Adopted Parameters for the Outer Disk}

The flux across (sub-)millimeter wavelengths provides an indication of the mass contained within the dust component of protoplanetary disks (e.g., Hildebrand 1983; Beckwith et al. 1990) as the emission is optically thin. In preliminary modeling with TORUS, we found that the (sub-)millimeter portion of the SED was reasonably well fit using a total disk mass of $0.20 M_{\odot}$, assuming a radially invariant gas-to-dust ratio of 100:1. As the dust grain prescription we adopt in our TORUS modeling is rather simplistic (see previous subsection), we acknowledge that this assessment of the disk mass is likely unrealistic. For instance, the adoption of a different grain size distribution or inclusion of another grain species with a different mass density and optical properties would affect the inferred value (cf. Wood et al. 2002).

The radial extent of the disk around HD 142666 has been constrained from VLT/NACO imaging (Garufi et al. 2017) and through analysis of rotationally broadened emission lines of gaseous species in the outer disk regions (Dent et al. 2005). In both cases, a value of $\sim 60$ au was indicated, assuming a distance to HD 142666 of $150 \mathrm{pc}$. This value is also consistent with the 65 au found recently by Rubinstein et al. (2018) from ALMA band 6 continuum observations. Our preliminary TORUS models showed that an outer disk radius of $R_{\text {out }}=60$ au provided a good fit to the long wavelength portion of the SED. This value was adopted in all our TORUS models.

\section{Results from Geometric Modeling}

As outlined in Section 3.1, an independently assessed $F_{\star}$ estimate was used to avoid degeneracies associated with using geometric models to simultaneously fit $F_{\star}$ and the characteristic size of the NIR-emitting region. To estimate $F_{\star}$ at $1.67 \mu \mathrm{m}(\mathrm{H}-$ band) and $2.13 \mu \mathrm{m}$ ( $K$-band), estimates of the stellar effective temperature, $T_{\text {eff }}$; surface gravity, $\log g$; and metallicity, $\log Z$, of HD 142666 were retrieved from the literature (see Table 4). Using the Python package PYSYNPHOT (STScI Development
Team 2013) and the "minimize" function of the Python LMFIT library (Newville et al. 2014), the corresponding Kurucz (1979) model atmosphere was compared to Johnson-Cousins $B V I_{\mathrm{C}}$ photometry to assess the $V$-band extinction, $A_{V}$, and the stellar radius, ${ }^{9} R_{\star}$. As HD 142666 displays flux variations at optical and NIR wavelengths (e.g., Makarov et al. 1994; cf. Section 6.2), we ensured that the $B V I_{\mathrm{C}}$ photometries were obtained contemporaneously. The reddening law of Cardelli et al. (1989) with a total-to-selective extinction $R_{V}=5.0$ was adopted based on previous analyses of Herbig Ae/Be stars by Hernández et al. (2004) and Manoj et al. (2006). The fitting procedure uses the differential evolution method, which is less susceptible to regions of local minima than, e.g., the Levenberg-Marquardt method. The values of $R_{\star}$ and $A_{V}$ found in the fitting process were then combined with the values of $T_{\text {eff }}, \log g, d$, and $\log Z$ to estimate $F_{\star, H}$ and $F_{\star, K}$. The best-fit values are presented in Table 4 .

These results were used to determine self-consistent estimates for the remaining stellar parameters required as inputs for the TORUS radiative transfer models. The stellar luminosity, $L_{\star}$, was estimated from the $V$-band magnitude and extinction using $T_{\text {eff }}$-dependent bolometric corrections taken from Table 5 of Pecaut \& Mamajek (2013) and a value of 4.755 mag for the bolometric magnitude of the Sun (Mamajek 2012). The stellar mass, $M_{\star}$, was estimated by comparing $T_{\text {eff }}$ and $L_{\star}$ to Siess et al. (2000) pre-main-sequence evolutionary models with $Z=0.02$ (without convective overshooting). These values are also presented in Table 4.

$\mathrm{PS}+\mathrm{R}$ models were fit to the visibilities obtained with CLASSIC and CLIMB before repeating the process with the shorter baseline PIONIER ( $H$-band) or KI $(K$-band) data included. In each case, the $H$ - and $K$-band data were fit separately. The stellar flux contribution remained fixed at the values in Table 4. The resulting best-fit parameters (and corresponding $\chi_{\mathrm{r}}^{2}$ ) for each model are displayed in Table 5. The observed $\mathrm{H}$ - and $\mathrm{K}$-band visibilities are compared to those of the best-fit models (gray solid lines) in Figures 3 and 4, respectively.

The $\mathrm{PS}+\mathrm{R}$ model suggests $i \sim 53^{\circ}-61^{\circ}$ and $\mathrm{PA}_{\text {major }} \sim$ $155^{\circ}-166^{\circ}$, depending on the data set. Inclusion of the shorter baseline data favors slightly lower inclinations in $K$-band and reduces the uncertainty range on our estimates of $i$ and $\mathrm{PA}_{\text {major }}$ across both wavebands. These values for $i$ and $\mathrm{PA}_{\text {major }}$ agree well with previous analyses of NIR and MIR interferometric data $\left(30^{\circ}<i<60^{\circ}\right.$ and $\mathrm{PA}_{\text {major }} \sim 170^{\circ}$ Vural et al. 2014; Lazareff et al. 2017) together with the recent VLT/ NACO imaging of Garufi et al. (2017), who found a preference for an inclined disk with $\mathrm{PA}_{\text {major }}$ along a north-south direction.

According to the $\mathrm{PS}+\mathrm{R}$ model-fitting results, the $H$-bandemitting region has an effective radius of $\sim 1.44-1.61$ mas $(\sim 0.22-0.24$ au at $150 \mathrm{pc})$. Assuming this region is associated

\footnotetext{
9 The radius enters the fit through the scaling factor, $\left(d / R_{\star}\right)^{2}$, which arises from the Kurucz (1979) model being in units of surface flux.
} 
Table 5

Results from PS $+\mathrm{R}$ Model Fits to $H$ - and $K$-band Visibilities

\begin{tabular}{|c|c|c|c|c|c|}
\hline Data Set & $R$ (mas) & $i\left(^{\circ}\right)$ & P.A. $\left({ }^{\circ}\right)$ & $F_{\star}$ & $\chi_{\mathrm{r}}^{2}$ \\
\hline \multicolumn{6}{|c|}{$H$-band } \\
\hline CLIMB & $1.61 \pm 0.08$ & $57.1 \pm 5.1$ & $163 \pm 14$ & 0.61 (fixed) & 21.97 \\
\hline \multirow[t]{2}{*}{ CLIMB+PIONIER } & $1.44 \pm 0.05$ & $55.5 \pm 2.1$ & $155 \pm 2$ & 0.61 (fixed) & 11.41 \\
\hline & $1.58 \pm 0.09$ & $57.1 \pm 1.7$ & $159 \pm 3$ & $0.64 \pm 0.03$ & 10.71 \\
\hline \multicolumn{6}{|c|}{$K$-band } \\
\hline & $1.46 \pm 0.06$ & $55.0 \pm 3.3$ & $156 \pm 4$ & $0.46 \pm 0.06$ & 21.03 \\
\hline \multirow[t]{2}{*}{ CLIMB +CLASSIC $+\mathrm{KI}$} & $1.28 \pm 0.04$ & $57.4 \pm 3.0$ & $162 \pm 4.2$ & 0.35 (fixed) & 34.10 \\
\hline & $1.50 \pm 0.07$ & $52.9 \pm 2.6$ & $164 \pm 5$ & $0.46 \pm 0.06$ & 19.98 \\
\hline
\end{tabular}
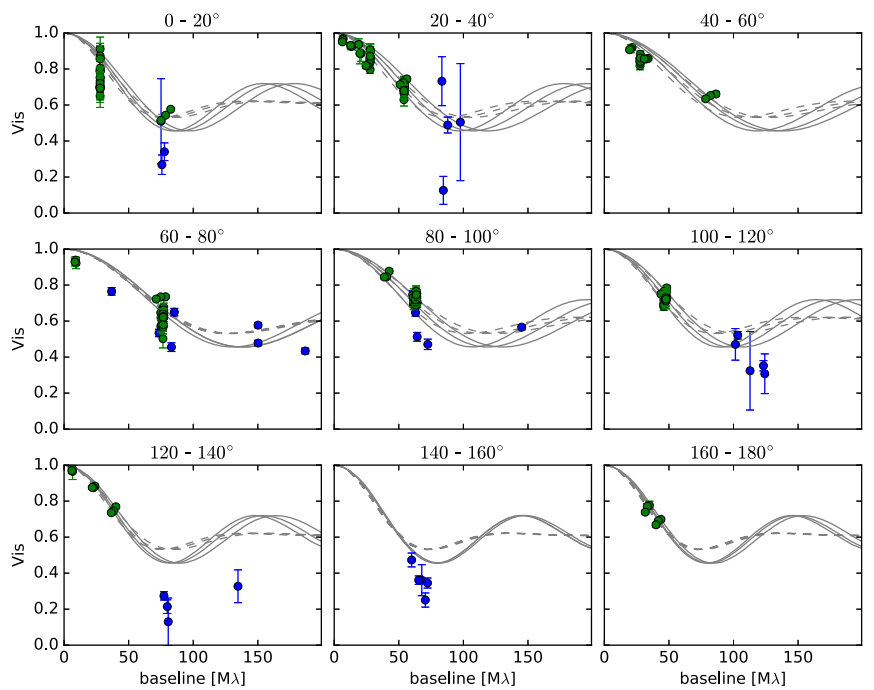

Figure 3. $\mathrm{PA}_{\text {base }}$-separated $H$-band visibilities plotted against spatial frequency. Gray solid lines represent the best-fit PS $+\mathrm{R}$ (with fixed $F_{\star, H}$ ) model visibility curves for the CLIMB+PIONIER data set (see Table 5). Gray dashed lines represent the best-fit PS + SR model visibility curves for the same data set (see Table 6). Three model curves are plotted in each panel, corresponding to steps of $10^{\circ}$ in $\mathrm{PA}_{\text {base }}$. The range of $\mathrm{PA}_{\text {base }}$ included in each figure window is listed above each window. Data points are colored as in Figure 1.

with dust sublimation, we use the Whitney et al. (2004) temperature-radius relation,

$$
R_{\mathrm{sub}}=R_{\star}\left(\frac{T_{\mathrm{sub}}}{T_{\mathrm{eff}}}\right)^{-2.1},
$$

to infer a sublimation temperature, $T_{\text {sub }} \sim 1750-1820 \mathrm{~K}$. Typically quoted values of $T_{\text {sub }}$ for silicate grains vary between $\sim 1500$ and $1800 \mathrm{~K}$ (Pollack et al. 1994), indicating that the $\mathrm{H}$-band emission is consistent with arising from the silicate dust sublimation rim. This value of $R$ is larger than the inferred value of 1.3 mas from Vural et al. (2014), but we note that those authors also adopt a lower value of $F_{\star, H}(0.53)$. The degeneracy between the stellar flux contribution and characteristic size of the emitting region found through geometric modeling is well known (see Lazareff et al. 2017 for a discussion) and is likely responsible for these differences.

Interestingly, at first glance, our $\mathrm{PS}+\mathrm{R}$ model fitting suggests that the $K$-band emission from HD 142666 traces material interior to the $H$-band-emitting region. This is counterintuitive as longer wavelength emission traces cooler
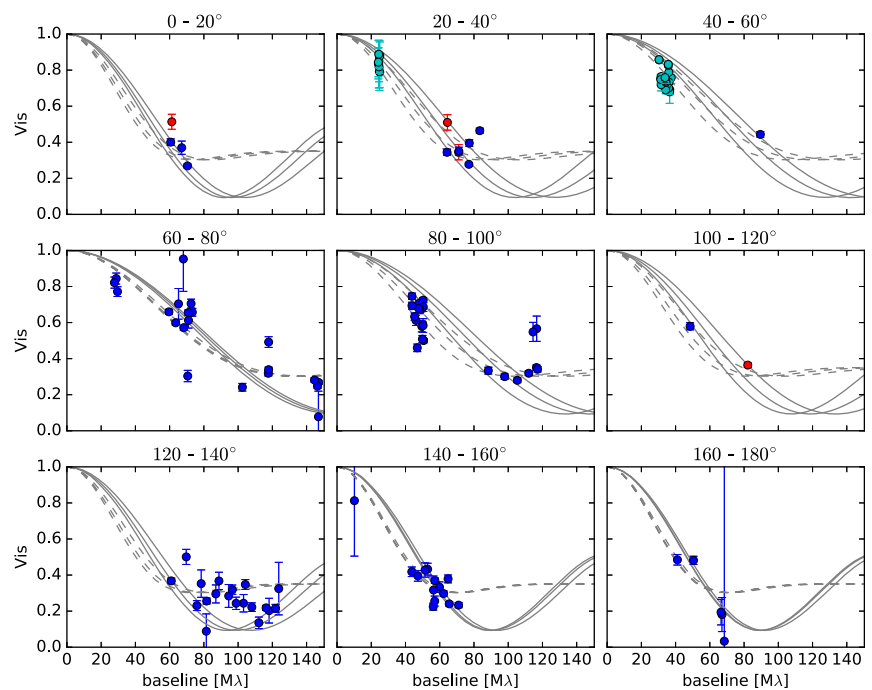

$140-160^{\circ}$

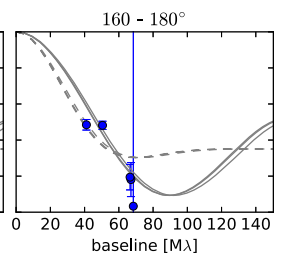

Figure 4. As Figure 3 but for the $K$-band. Gray solid lines represent the best-fit $\mathrm{PS}+\mathrm{R}$ (with fixed $F_{\star, K}$ ) model visibility curves for the CLIMB +CLASSIC $+\mathrm{KI}$ data set (see Table 5), while gray dashed lines correspond to those of the bestfit PS+SR model (see Table 6).

material. If stellar radiation is the dominant heating mechanism, the cooler, $K$-band emission should emerge from disk radii larger than those where warmer, $H$-band emission emerge. However, under closer inspection, this is more likely to be a result of the poorer fit provided by the $\mathrm{PS}+\mathrm{R}$ model to the $K$-band visibilities compared to those at $H$-band wavelengths. We investigated alternative models in an attempt to improve the fit. First, we relaxed the constraint on $F_{\star}$, allowing it to vary between 0 and 1 in the fitting process. ${ }^{10}$ Second, we adopted PS + SR models with $F_{\star}$ fixed at the values in Table 4 . The resulting best-fit values and $\chi_{\mathrm{r}}^{2}$ for these alternative models are presented in Tables 5 and 6 , respectively.

From Table 5, we can see that the fit is improved in all cases when the constraints on $F_{\star}$ are lifted. For the $H$-band emission, the fitted $F_{\star}$ values are consistent with the values adopted in our prior fitting within their uncertainties: $0.57 \pm 0.07$ (CLIMB) and $0.65 \pm 0.04$ (CLIMB +PIONIER) compared with the value of 0.61 found via SED fitting. For the $K$-band emission, both data sets reveal a preferred value of $0.46 \pm 0.06$ over the value of 0.35 found via SED fitting. HD 142666 exhibits variability across optical and NIR wavelengths (Meeus

\footnotetext{
${ }^{10}$ The total emission remained at 1 so the circumstellar emission provided a flux contribution of $1-F_{\star}$.
} 
Table 6

Results from PS+SR Model Fits to $H$ - and $K$-band Visibilities

\begin{tabular}{lccrr}
\hline \hline Data Set & $R$ (mas) & $i\left(^{\circ}\right)$ & P.A. $\left({ }^{\circ}\right)$ & FWHM (mas) \\
\hline CLIMB & \multicolumn{5}{c}{$\chi_{\mathrm{r}}^{2}$} \\
CLIMB+PIONIER & $1.78 \pm 0.09$ & $57.2 \pm 5.0$ & $165 \pm 14$ & $<2 \times 10^{-4}$ \\
\hline & $1.59 \pm 0.04$ & $56.7 \pm 1.9$ & $159 \pm 2$ & $0.60 \pm 0.09$ \\
\hline CLIMB+CLASSIC & \multicolumn{3}{c}{-band } & 9.54 \\
CLIMB+CLASSIC+KI & $1.51 \pm 0.08$ & $62.9 \pm 3.6$ & $153 \pm 3$ & $0.87 \pm 0.09$ \\
\hline
\end{tabular}

et al. 1998; Zwintz et al. 2009), and these discrepancies in $F_{\star}$ are consistent with the intrinsic $H$ - and $K$-band variability of 0.14 and $0.30 \mathrm{mag}$, respectively. At the same time, an increase (decrease) in $F_{\star}$ coincides with an increase (decrease) in $R$, highlighting the degeneracy that exists when fitting $R$ and $F_{\star}$ simultaneously. Without the constraints on $F_{\star}$, we see that the characteristic radius of the $H$ - and $K$-band-emitting regions is consistent within the bootstrapped errors.

A further reduction in $\chi_{\mathrm{r}}^{2}$ is provided by the PS $+\mathrm{SR}$ models (dashed gray lines in Figures 3 and 4). The PS $+\mathrm{R}$ model prescribes the NIR-emitting region as a central star with circumstellar emission provided by a vertical disk rim. In comparison, the circumstellar component of the PS + SR model emulates a more rounded rim in which the emitting region is more spatially extended. The better fit provided by the PS + SR model over the PS $+\mathrm{R}$ model suggests that the inner rim of the disk of HD 142666 is not well approximated by a vertical wall. This is consistent with previous studies of other Herbig $\mathrm{Ae} / \mathrm{Be}$ stars (e.g., Tannirkulam et al. 2007; Kraus et al. 2009; McClure et al. 2013). As with the PS $+\mathrm{R}$ models, we see that the characteristic radius of the $H$-band-emitting region found via the PS+SR model fitting is consistently larger than that of the $K$-band emission though the two are roughly consistent within their estimated uncertainties: $1.59 \pm 0.04$ mas (CLIMB+PIONIER; $H$-band) compared with $1.50 \pm 0.08$ mas (CLIMB + CLASSIC + KI; $K$-band). Furthermore, the FWHM of the Gaussian component used to convolve the ring in these models is larger in the $K$-band than in the $H$-band, suggesting that the $K$-band emission may originate over a broader range of disk annuli.

\section{Results from TORUS Radiative Transfer Modeling}

In light of our geometric modeling results, we adopted $i=58^{\circ}$ and $\mathrm{PA}_{\text {major }}=160^{\circ}$ throughout our TORUS modeling for the computation of model SEDs and images. Figure 5 shows the distribution of squared visibilities $\left(V^{2}\right)$ as a function of effective spatial frequency, which accounts for the change in resolution across the $u v$ plane due to this inferred viewing geometry. With inclination effects accounted for, the vertical scatter in $V^{2}$ at each effective spatial frequency is assumed to arise due to the combined effects of calibration uncertainties and temporal variability (see Section 6.2).

When extracting $\phi_{\mathrm{CP}}$ from the TORUS model images, we orient the disk such that its northeastern portion is the far side (and, thus, the brighter side) of the disk. This is based on the asymmetric brightness distribution seen in the VLT/NACO polarimetry (Garufi et al. 2017).
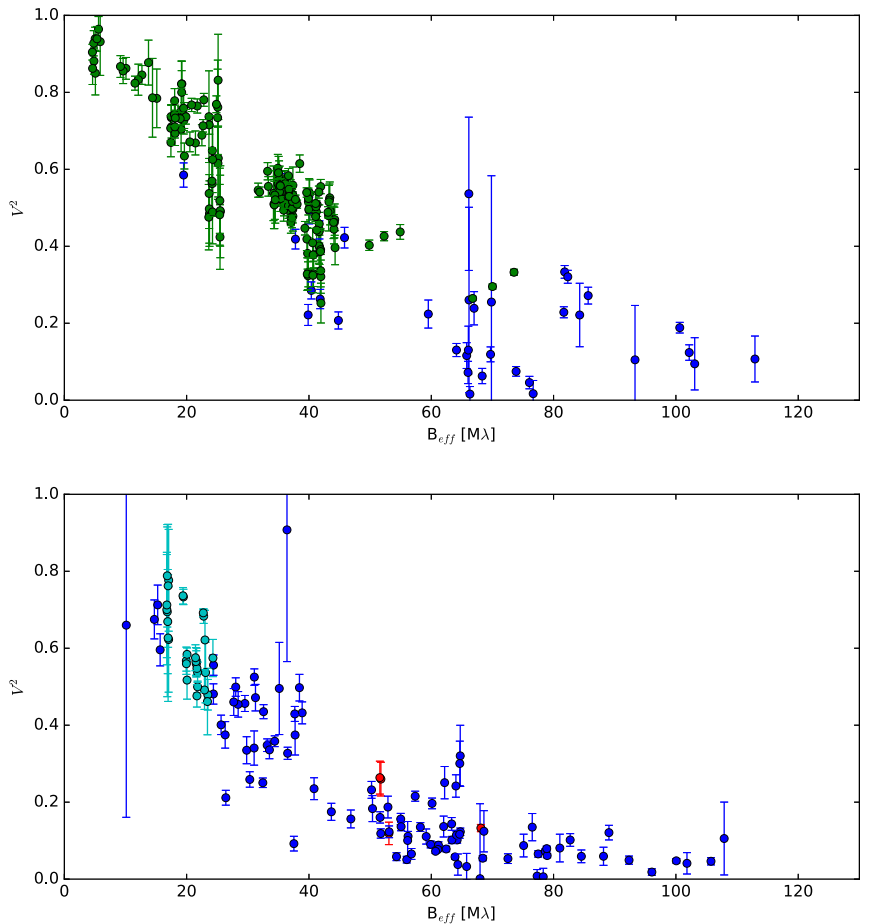

Figure 5. Distribution of $H$-band (top) and $K$-band (bottom) squared visibilities with respect to the effective spatial frequency, computed using $i=58^{\circ}$ and $\mathrm{PA}_{\text {major }}=160^{\circ}$. Data points are colored as in Figure 1.

Table 7

Converged Structure of TORUS Models Computed at Disk Inclinations of $58^{\circ}$ with Vertical Hydrostatic Equilibrium Imposed and Their Respective Stellar Contribution to the Total Model $H$ - and $K$-band Fluxes

\begin{tabular}{lcccc}
\hline \hline Model & $h_{0, \text { gas }}(\mathrm{au})$ & $\beta$ & $F_{\star, H}$ & $F_{\star, K}$ \\
\hline S:small & 4.6 & 1.14 & 0.85 & 0.68 \\
S:large & 5.0 & 1.17 & 0.67 & 0.50 \\
THM07 & 4.7 & 1.18 & 0.75 & 0.62 \\
\hline
\end{tabular}

\subsection{Models Imposing Vertical Hydrostatic Equilibrium}

We first computed the S:small, S:large, and THM07 models with vertical hydrostatic equilibrium established. In each case, the disk temperature and density structure converged after three iterations. We used Equation (3) to determine an approximate value of $\beta$ for the converged disk structure and present these alongside the values of $h_{0, \text { gas }}, F_{\star, H}$, and $F_{\star, K}$ in Table 7 . The SEDs computed for each model were reddened and are compared to the observed SED in Figure 6. A relative dearth of NIR flux up to $\sim 3 \mu \mathrm{m}$ combined with a relative excess of flux over $\sim 3-10 \mu \mathrm{m}$ is provided by the $\mathrm{S}$ :small model (solid 


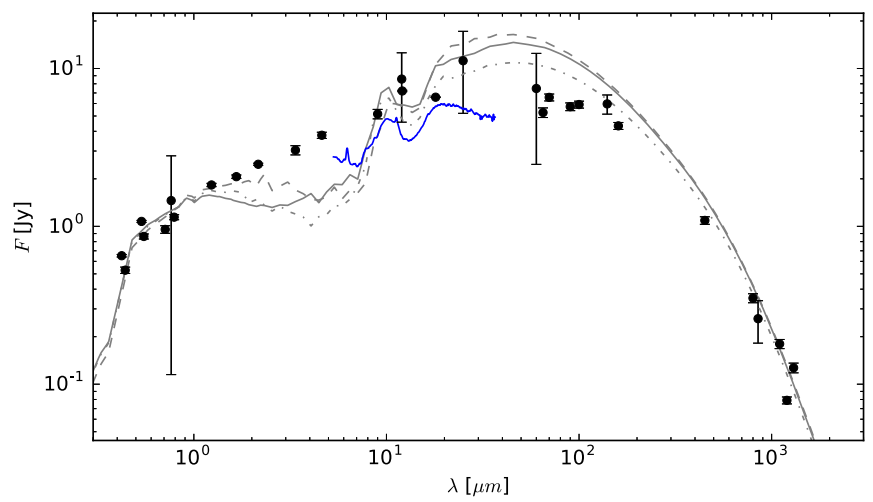

Figure 6. SEDs for our TORUS models with vertical hydrostatic equilibrium enforced. Photometric data are shown as black points while the Spitzer spectrum is represented by a solid blue line. Gray lines correspond to the reddened TORUS model SEDs computed at $i=58^{\circ}$ (solid line: S:small; dashed line: S:large; dotted-dashed line: THM07).

gray line) compared with the S:large and THM07 models (dashed and dotted-dashed lines, respectively). Interestingly, the models including larger grains produce noticeably different SED shapes across NIR wavelengths: although the flux across the $H$ - and $K$-bands is underestimated in both cases, the S:large model produces NIR flux levels closest to those observed. These differences arise due to differences in the size of the disk area that directly intercepts stellar radiation in the innermost disk regions. The inclusion of larger grains extends the inner edge of the disk to smaller radii, as already discussed in, for example, Monnier \& Millan-Gabet (2002), Isella \& Natta (2005), Tannirkulam et al. (2007), Kama et al. (2009), and McClure et al. (2013). The rim curvature provided by the THM07 model is also shallower and more extended than that from the S:large model. As such, if we consider the surface layers of the disk behind the sublimation rim, the directly illuminated disk area between disk annuli, $r$, and $r+\delta r$ will be smaller in the THM07 model than in the S:large model. As more optically thick disk material exist at hotter temperatures, this produces a larger NIR flux-emitting disk area.

In all three cases, our assumption that all the circumstellar NIR emission arises from a disk in vertical hydrostatic equilibrium leads to a poor SED fit. To better reproduce the observed SED, our models require more NIR emission at the expense of FIR emission. This discrepancy has previously been seen in both radiation hydrodynamic and radiation hydrostatic model fits to the SEDs of other Herbig AeBe stars (Mulders \& Dominik 2012; Flock et al. 2016). Turbulence associated with, for example, magnetorotational instability (MRI) (Turner et al. 2014; Flock et al. 2017) and/or the presence of magnetospheric or photoevaporative disk winds (Alexander \& Armitage 2007; Bans \& Königl 2012) could contribute to lifting optically thick material above the disk scale heights predicted by our hydrostatic models. In addition, the presence of any optically thick gaseous material existing interior to the dust sublimation rim (Tannirkulam et al. 2008a, 2008b) would affect the temperature structure of the dusty disk. As our CHARA interferometry does not reveal a bounce in the visibilities (Figure 5), we are unable to comment on whether optically thick material interior to the sublimation rim contributes to the NIR flux. The extension of current optical interferometry facilities such as the CHARA Array to longer operational baselines and/or the construction of longer baseline optical interferometers equipped with NIR detectors (e.g.,
Planet Formation Imager; Kraus et al. 2014) are essential for investigating whether NIR continuum emission also arises interior to the silicate sublimation rim in disks of later type Herbig Ae stars and their low-mass counterparts, the T Tauri stars. The introduction of a dusty disk wind is also beyond the scope of this paper, and we defer this to future study. Instead, in the subsections that follow, we focus on whether turbulenceinduced scale height inflation is able to simultaneously fit the observed SED and interferometry of HD 142666.

To artificially emulate scale height inflation in the inner disk, we computed a series of grids of TORUS models without establishing vertical hydrostatic equilibrium. Each model grid was computed at a range of gas disk scale heights $\left(h_{0, \text { gas }}=6\right.$, $7,8,9,10,11 \mathrm{au})$ and flaring parameters $(\beta=1.05,1.06,1.07$, $1.08,1.09,1.10)$ while the stellar parameters, disk mass, and outer disk radius each remained fixed (see Table 4).

\subsection{Small Grain Models}

Over the range of $h_{0, \text { gas }}$ and $\beta$ probed, none of our S:small models were able to reproduce the observed SED across the full wavelength range. In the top left panel of Figure 7, the SED of HD 142666 is compared to the reddened SEDs of the two bestfitting S:small models. The S:small model with $h_{0 \text {,gas }}=7 \mathrm{au}$ and $\beta=1.09$ (dashed gray line) provides the best fit to the SED over the full wavelength range but clearly provides insufficient NIR flux. To fit the NIR portion of the SED, a greater scale height was required: the $h_{0}=10$ au and $\beta=1.06$ model (solid gray line) provides the best fit across this wavelength range while still reproducing the SED longward of $\sim 100 \mu \mathrm{m}$. However, this latter model clearly overestimates the MIR-to-FIR flux.

As the NIR flux is well approximated by the S:small model with $h_{0}=10$ au and $\beta=1.06$, we examined the visibilities and $\phi_{\mathrm{CP}}$ for this model to inspect the rim position and shape. These are displayed in the remaining panels of Figure 7. Here, as in Figures 3 and 4, the different panels show the visibilities measured along different baseline position angles, $\mathrm{PA}_{\text {base }}$. In addition to providing too much flux across MIR-to-FIR wavelengths, we see in the bottom panels of Figure 7 that the first lobe of the model visibility curves drop more rapidly at short baselines than measured by the data. As such, optically thick material is required to exist interior to the silicate dust sublimation rim location predicted by S:small models. This is consistent with results from analyses of NIR size-luminosity relations for Herbig Ae stars in which the size of the NIRemitting region is controlled by the sublimation of larger grains ( $\sim 1 \mu \mathrm{m}$ in size; e.g., Monnier \& Millan-Gabet 2002).

\subsection{Models Invoking Grain Growth}

Figures 8 and 9 show the SED, visibilities, and $\phi_{\mathrm{CP}}$ of the best-fitting S:large and THM07 models, respectively. The $\chi_{\mathrm{r}}^{2}$ fits to the $\operatorname{SED}\left(\chi_{\mathrm{r}, \mathrm{SED}}^{2}\right)$, visibilities $\left(\chi_{\mathrm{r}, \mathrm{vis}}^{2}\right)$, and $\phi_{\mathrm{CP}}\left(\chi_{\mathrm{r}, \mathrm{CP}}^{2}\right)$ are presented in Table 8 . Models invoking grain growth to micron sizes clearly provide an improved fit to the SED and visibilities compared to the S:small models we explored. The best-fitting S:large and THM07 models are both able to provide a reasonable estimate of the fluxes in the SED across the full range of wavelengths probed. Though the SEDs provided by the S:large and THM07 models in Figures 8 and 9 are broadly consistent with one another, the S:large model is able to 

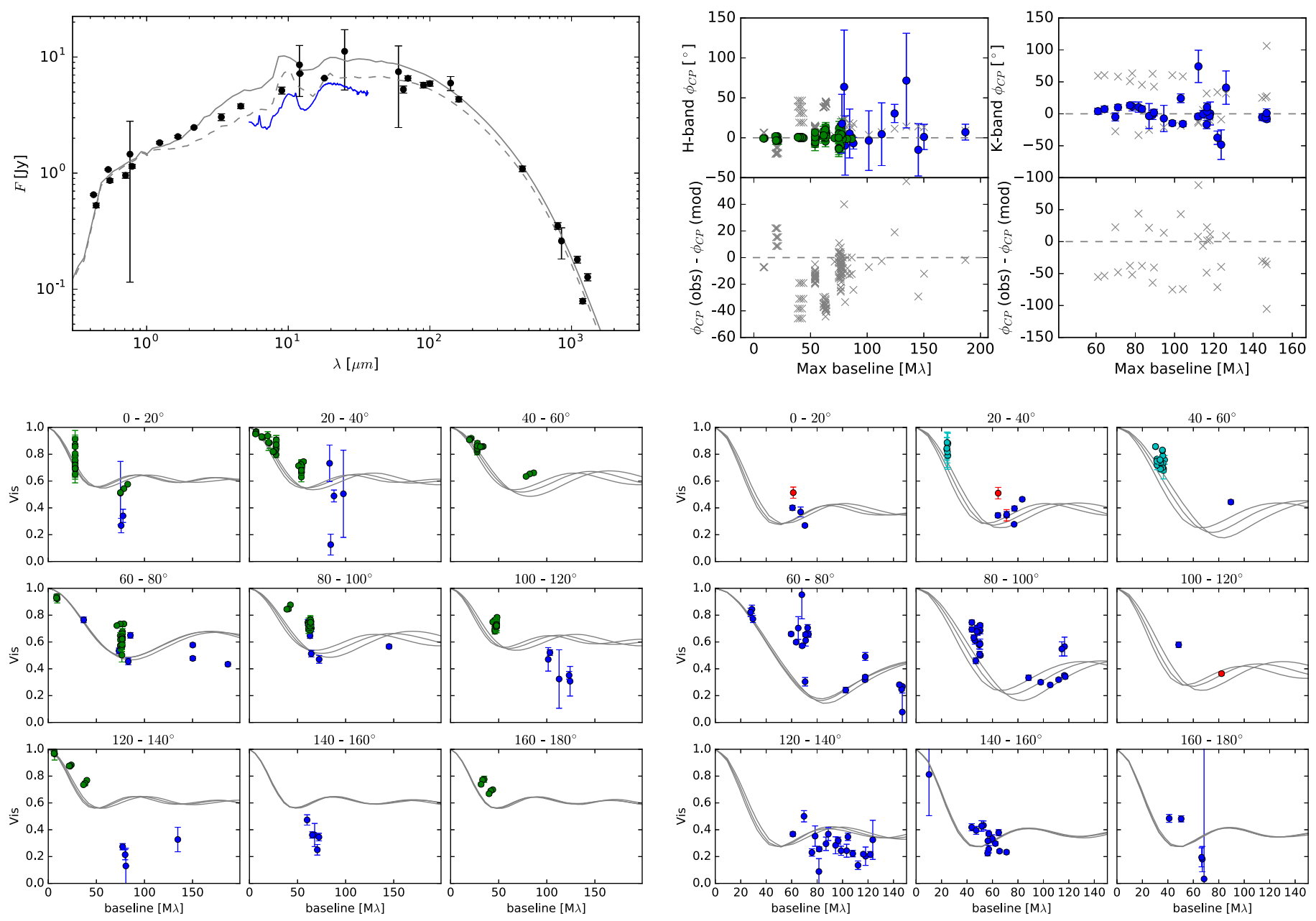

Figure 7. Summary plots for S:small model providing the best fit to the observed SED across the NIR $\left(h_{0}=7\right.$ au, $\left.\beta=1.09\right)$. Top left: as Figure 6 but comparing this best-fit model (solid gray line) to the S:small model with $h_{0}=10$ au and $\beta=1.06$ (dashed gray line). Top middle and top right: $H$ - and $K$-band $\phi_{\mathrm{CP}}$ (top) and the residuals (bottom), respectively. Both are displayed as a function of the maximum spatial frequency probed by the closed triangle of baseline vectors. Bottom rows: visibilities as a function of spatial frequency, separated by $\mathrm{PA}_{\mathrm{base}}$. As in Figure 3, three model visibility curves are plotted in each panel corresponding to $10^{\circ}$ steps in $\mathrm{PA}_{\text {base }}$. The nine panels on the left-hand side correspond to the $H$-band data while those on the right are for the $K$-band data. Data in the visibility and $\phi_{\mathrm{CP}}$ plots are colored as in Figure 1.

reproduce the general shape of the Spitzer spectrum out to $\sim 12 \mu \mathrm{m}$ better than the THM07 model.

The scale height and flaring parameters of the best-fitting models are broadly consistent: $h_{0 \text {,gas }}=7$ au and $\beta=1.09$ for S:large versus $h_{0, \mathrm{gas}}=8$ au and $\beta=1.09$ for THM07. The S:small model, which provided the best fit across the full wavelength range probed by the observed SED (while underestimating the NIR flux; see dashed gray line in the top left panel of Figure 7), also had $h_{0 \text {,gas }}=7$ au and $\beta=1.09$. The differences in the scale heights required for the different models to produce the same NIR flux is consistent with what we saw for the models invoking vertical hydrostatic equilibrium (Section 5.1) whereby the different rim curvature prescriptions give rise to inner rims with varying radial extents. This is shown more clearly in Figure 10: the rim produced by the $\mathrm{S}$ :small model is located farther from the star than that produced by the S:large and THM07 models. The curvature of the rim produced by the THM07 model is also sharper than that produced by the S:large model. As a result, the NIR flux arises from a smaller range of disk radii than the S:large model.

The best-fitting S:large and THM07 models provide similarly good fits to the observed $H$ - and $K$-band visibilities with the S:large model providing a marginally better fit to the observed visibilities than the THM07 model (see Table 8). The first lobe of the model visibility curves is in good agreement with the data across most $\mathrm{PA}_{\text {base}}$, suggesting that the location of the inner disk rim of HD 142666 is consistent with the silicate sublimation region predicted by the models invoking grain growth to micron sizes. In Figure 10, we see that the THM07 models predict a rim location that is slightly more extended than that of the S:large models while the $\mathrm{S}$ :large model is able to provide more flux in the southwest portion of the disk.

Upon closer inspection, the visibilities and $\phi_{\mathrm{CP}}$ in Figures 8 and 9 indicate an additional complexity in the circumstellar component of the NIR emission which remains unexplained in our suite of models. In the visibility plots, the models appear underresolved compared to the data along the apparent disk minor axis $\left(60^{\circ}-80^{\circ} \mathrm{PA}_{\text {base }}\right.$ panels $)$, while the often significant $\left(\sim 50^{\circ}-100^{\circ}\right) \phi_{\mathrm{CP}}$ signals predicted by the models are not present in the data. These discrepancies indicate the presence of additional material along the disk minor axis interior to the skyprojected location of the dust sublimation rim predicted by our models as well as a more centrosymmetric brightness distribution. We discuss this further in Section 6.2. 

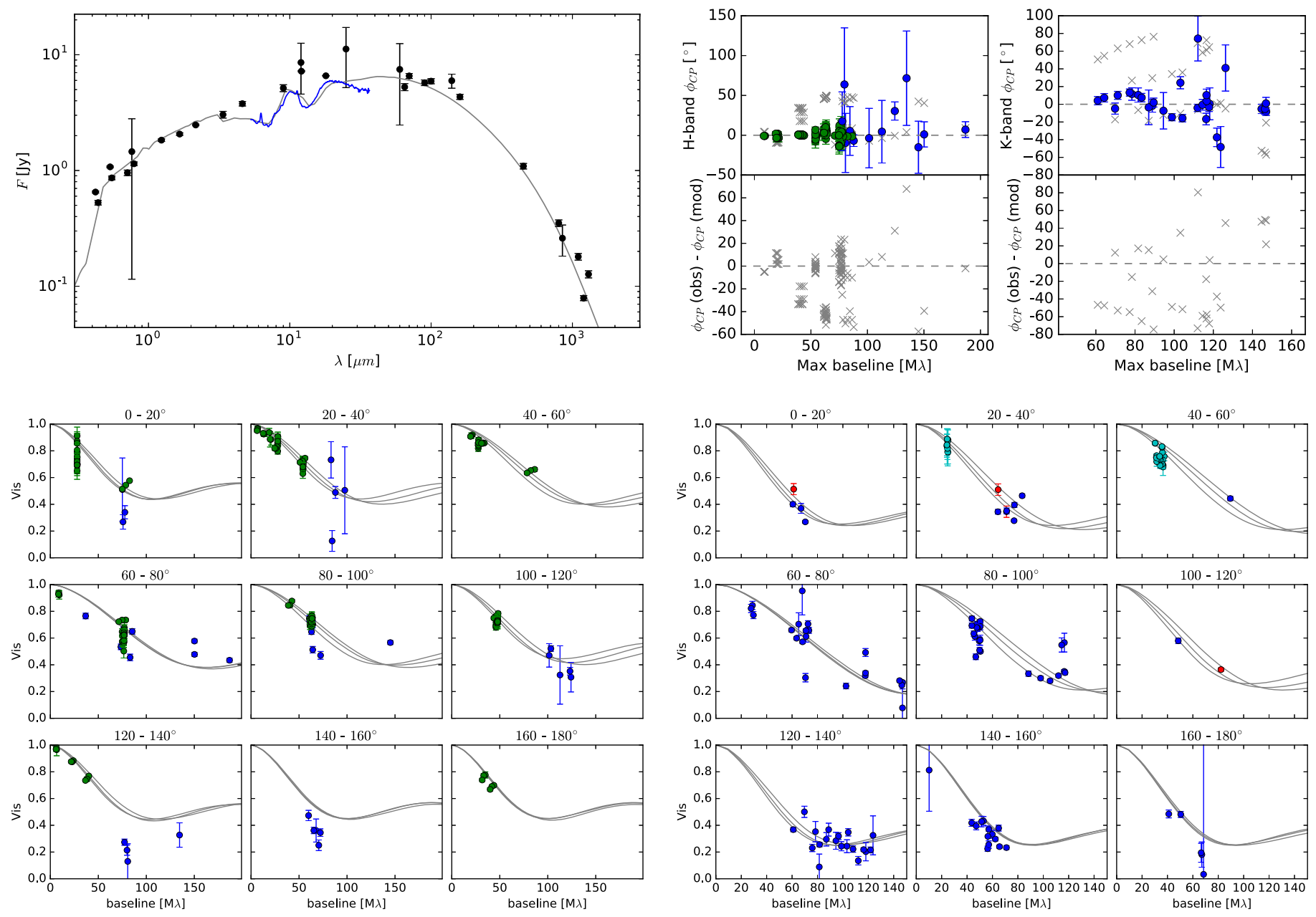

Figure 8. As Figure 7 but for the S:large model providing the best fit to the observed SED $\left(h_{0, \text { gas }}=7\right.$ au and $\left.\beta=1.09\right)$.

\section{Discussion}

\subsection{Grain Growth in the Disk of HD 142666 and the Inner Rim Location}

The results presented in Section 5 indicate that models in which the inner disk rim is dominated by small $(0.1 \mu \mathrm{m})$ grains are incompatible with the SED and NIR interferometry obtained for HD 142666. Instead, models invoking the growth of dust grains to micron sizes provide improved fits to the observations. These results support those of van Boekel et al. (2003), who, in their analysis of the shape and strength of the silicate feature in the Spitzer spectrum of HD 142666, found strong evidence for growth from $0.1 \mu \mathrm{m}$ to $2.0 \mu \mathrm{m}$ for grains with a mass ratio of 1:1.54 in favor of large grains. As MIR emission arises from the disk surface layers, and larger grains are expected to settle to lower scale heights in the disk (Testi et al. 2014), the dominance of micron-sized grains in the disk midplane was anticipated to be even more pronounced. Our results support this idea as the models invoking the presence of larger, micron-sized grains (S:large model with $h_{0, \text { gas }}=7$ au and $\beta=1.09$ and THM07 model with $h_{0 \text {,gas }}=8$ au and $\beta=1.09$ ) are able to simultaneously reproduce the NIR portion of the SED, the shape and flux of the Spitzer spectrum, and the observed $H$ - and $K$-band visibilities.

The lower $\chi_{\mathrm{r} \text {,vis }}^{2}$ provided by the S:large model fit to the visibilities compared to the THM07 model (see Table 8) further suggests that the inner disk rim of HD 142666 is more consistent with models invoking a gas-density-dependent dust sublimation temperature (e.g., Isella \& Natta 2005) than with those invoking constant dust sublimation temperatures where rim curvature arises due to the relative abundance of different grain sizes (in $r$ and $z$ ) and their relative cooling efficiencies (e.g., Tannirkulam et al. 2007). However, it should be noted that using (i) grains $<1.2 \mu \mathrm{m}$ as the larger grains, (ii) a different size for the smaller grains, (iii) a different value for $h_{0 \text {,dust }}$ for the larger grains, and/or (iv) a different silicate sublimation temperature (see Table 3) would all affect the rim shape, location, and temperature structure predicted by the THM07 models. The parameters we adopted in our THM07 models were chosen for their consistency with the original Tannirkulam et al. (2007) study and a comprehensive evaluation of the impact of these variables is beyond the scope of this paper. However, our use of $1.2 \mu \mathrm{m}$ sized grains as the "large" grains should produce inner rim locations close to the lower limit allowed by the Tannirkulam et al. (2007) and Isella \& Natta (2005) models. This is because silicate grains larger than $\sim 1.3 \mu \mathrm{m}$ do not significantly contribute to the dust opacity and thus their inclusion would not make the rim any more compact (Isella \& Natta 2005).

Of the parameters explored herein, our best model (the S:large model with $h_{0, \text { gas }}=7$ au and $\beta=1.09$ ) produces a sublimation rim that remains optically thick down to within 0.17 au of the star (in the disk midplane). This is broadly consistent with the results of our geometric fitting (Section 4) in 

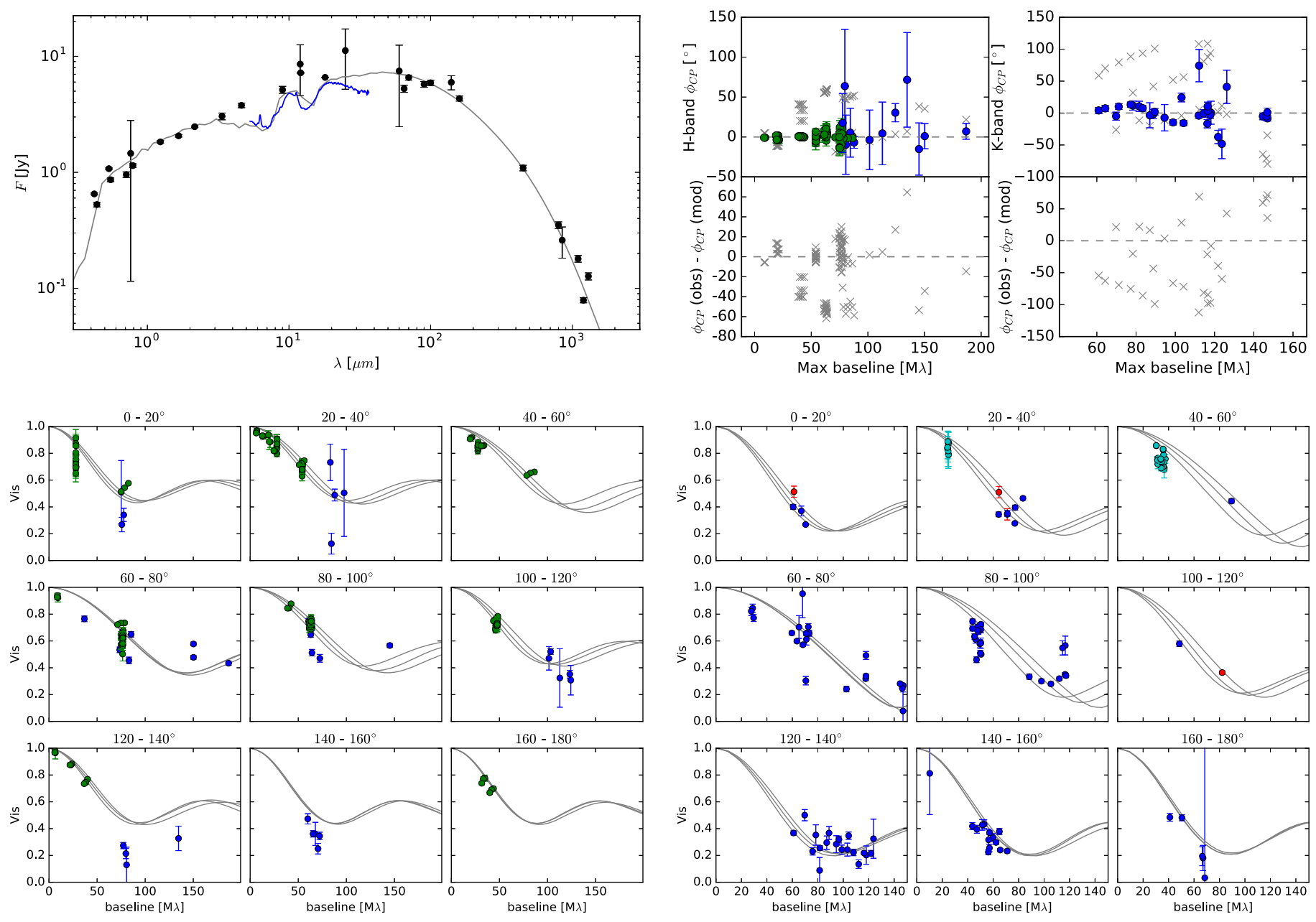

Figure 9. As Figure 7 but for the THM07 model providing the best fit to the observed SED across NIR wavelengths $\left(h_{0, \text { gas }}=8\right.$ au and $\left.\beta=1.09\right)$.

Table 8

Parameters of the Best-fitting TORUS Models without Vertical Hydrostatic Equilibrium Imposed (Adopting $i=58^{\circ}$ and $\mathrm{PA}_{\text {major }}=160^{\circ}$ ) and Their $\chi_{\mathrm{r}}^{2}$ Fits to the SED, Visibilities, and Closure Phases (362, 337, and 153 Degrees of Freedom, Respectively)

\begin{tabular}{lccrcr}
\hline \hline Model & $h_{0, \text { gas }}$ & $\beta$ & $\chi_{\mathrm{r}, \mathrm{SED}}^{2}$ & $\chi_{\mathrm{r}, \mathrm{vis}}^{2}$ & $\chi_{\mathrm{r}, \mathrm{CP}}^{2}$ \\
\hline S:small & 10 & 1.06 & 109,554 & 74.3 & 9.3 \\
S:large & 7 & 1.09 & 1086 & 14.4 & 16.3 \\
THM07 & 8 & 1.09 & 2029 & 22.1 & 18.2 \\
\hline
\end{tabular}

which the characteristic radii of the $H$ - and $K$-band-emitting regions were found to be $\sim 0.22-0.24$ au in both the PS $+\mathrm{R}$ and $\mathrm{PS}+\mathrm{SR}$ fits. These inner radii are lower than previously published estimates by Monnier et al. (2005) and Schegerer et al. (2013) based on short-baseline NIR and MIR visibilities ( $\sim 0.38-0.39$ au, accounting for differences in the adopted distance to HD 142666 ) but consistent with those in Vural et al. (2014, 0.19-0.23 au). However, we note that the adopted stellar parameters (including the stellar flux contribution) are consistent neither across these studies nor between these studies and our own. As discussed in Lazareff et al. (2017), the characteristic size of the emitting region and the circumstellar flux contribution are intrinsically linked in the visibility so it is understandable that differences in one parameter will lead to differences in the other when comparing studies.

\subsection{Indicators of Additional Complexity in the NIR-emitting Region}

Throughout our radiative transfer analysis (Section 5), we first required our TORUS models to reproduce the observed SED before assessing the fit to the interferometry. In this way, we assume that the disk in our TORUS models accounts for all of the NIR circumstellar flux. If additional NIR-emitting gaseous material exists interior to the sublimation rim (Tannirkulam et al. 2008a, 2008b) and/or a dusty outflow exists (Alexander \& Armitage 2007; Bans \& Königl 2012)— neither of which are accounted for in our models-they will also contribute to the observed $H$ - and $K$-band flux.

Additionally, in our geometric modeling (Section 4), we assumed that all of the circumstellar NIR flux could be fit using a Gaussian-smoothed ring model and, from this, estimated a disk major axis position angle and inclination of $160^{\circ}$ and $58^{\circ}$, respectively. While this viewing geometry agrees with previous assessments of the disk inclination $\left(40^{\circ}-60^{\circ}\right.$; Dominik et al. 2003; Vural et al. 2014; Lazareff et al. 2017; Rubinstein et al. 2018) and position angle $\left(\sim 140^{\circ}-180^{\circ}\right.$; Garufi et al. 2017; Rubinstein et al. 2018), indirect evidence for further model complexity is suggested in the visibility and $\phi_{\mathrm{CP}}$ residuals. As stated in Section 5.3, while the models invoking grain growth to micron sizes provide a good fit to the visibilities across a wide range of $\mathrm{PA}_{\text {base }}$, the model visibility curves appear underresolved compared to the data along the 

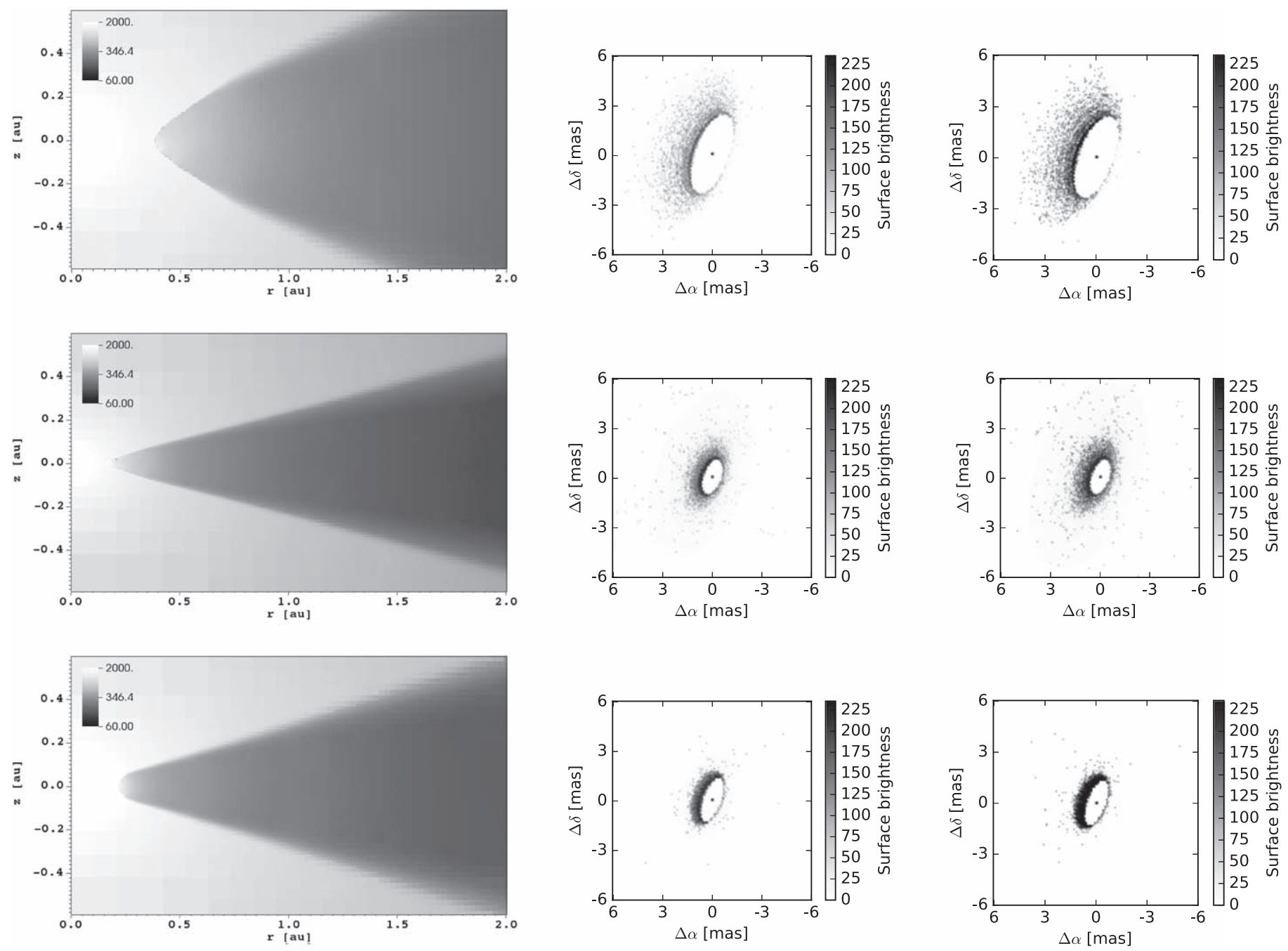

Figure 10. Cross section of the disk temperature profile (in Kelvin; left-hand panel) and model $H$ - and $K$-band images (middle and right-hand panels, respectively) output by TORUS for the S:small model with $h_{0, \text { gas }}=10$ au and $\beta=1.06$ (top row), the S:large model with $h_{0, \text { gas }}=7$ au and $\beta=1.09$ (middle row), and the THM07 model with $h_{0, \text { gas }}=8$ au and $\beta=1.09$ (bottom row).

apparent disk minor axis. In addition, the significant $\left(\sim 50^{\circ}-\right.$ $\left.100^{\circ}\right) \phi_{\mathrm{CP}}$ signals predicted by our best-fitting TORUS models are not present in the data, indicating that the true brightness distribution is more centrosymmetric.

Further indirect evidence of additional model complexity is found when considering the UX Ori-type phenomena displayed by HD 142666 (Meeus et al. 1998; Zwintz et al. 2009). This type of variability is associated with line-of-sight fluctuations in opacity and is typically attributed to circumstellar disk occultation (Grinin et al. 1991; Natta et al. 1997), although unsteady accretion (Herbst \& Shevchenko 1999) and/ or the existence of dusty outflows (Vinković \& Jurkić 2007; Tambovtseva \& Grinin 2008) have been proposed as alternative causes. As the disk- and outflow-based origins require intermediate-to-high disk inclinations for line-of-sight occultations to arise, and the inferred disk inclination of $58^{\circ}$ for HD 142666 is relatively low compared to the $\sim 70^{\circ}$ inferred for other UX Ori stars (VV Ser, KK Oph, and UX Ori itself; Pontoppidan et al. 2007; Kreplin et al. 2013, 2016), the photometric variability observed for HD 142666 may suggest that the disk is more inclined. Alternatively, the UX Ori variability may indicate that azimuthal and temporal variations in disk scale height exist.

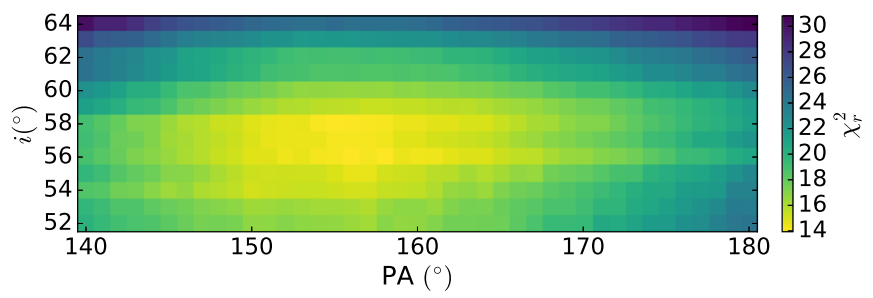

Figure 11. $\chi_{\mathrm{r}}^{2}$ map showing the comparative goodness of fit provided by the S:large model with $h_{0, \text { gas }}=7$ au and $\beta=1.09$ to the $H$ - and $K$-band visibilities using disk inclinations $52^{\circ} \leqslant i \leqslant 64^{\circ}$ and position angles $140^{\circ} \leqslant \mathrm{PA}_{\text {major }} \leqslant$ $180^{\circ}$.

To investigate whether the residuals in the interferometry fits could be reconciled solely by changing the disk viewing geometry, we explored whether a better fit to the visibilities could be achieved if our best-fit S:large TORUS model $\left(h_{0, \mathrm{gas}}=7\right.$ au and $\beta=1.09$ ) was observed at differing viewing geometries: $52^{\circ} \leqslant i \leqslant 64^{\circ}$ and $140^{\circ} \leqslant \mathrm{PA}_{\text {major }} \leqslant 180^{\circ}$. The resulting $\chi_{\mathrm{r}}^{2}$ map is shown in Figure 11. At the original viewing geometry $\left(\mathrm{PA}_{\text {major }}=160^{\circ}\right.$ and $\left.i=58^{\circ}\right)$, the model provides $\chi_{\mathrm{r}}^{2}=14.4$. Over the range of inclinations and position angles probed, the model with $i=58^{\circ}$ and $\mathrm{PA}_{\text {major }}=155^{\circ}$ provides the best fit to the visibilities but the improvement in $\chi_{\mathrm{r}}^{2}$ is small: 
$\chi_{\mathrm{r} \text { min }}^{2}=13.9$. This revised disk viewing geometry unsurprisingly still produces model visibilities that are underresolved along the apparent disk minor axis and model $\phi_{\mathrm{CP}}$ signals in excess of those observed. As such, the residuals in our TORUS model fitting cannot be explained simply by changing the viewing geometry and instead point to additional model complexity.

The disk models we have explored with TORUS assume azimuthal symmetry: we have not accounted for the possible presence of azimuthal variations of the disk scale height (i.e., disk warps). The disk of HD 142666 is not strongly flared (Section 5; cf. Meeus et al. 2001) and, as such, disk regions at large distances from the star are unlikely to provide line-ofsight stellar occultations when observed at an inclination of $58^{\circ}$ (see Figure 10). Assuming that optically thick material only exists exterior to the dust rim location predicted by the best-fit S:large TORUS model, a disk inclination of $58^{\circ}$ requires azimuthal scale height increases of around $40 \%$ in the inner disk for direct line-of-sight occultation. The periods of minimum brightness observed for HD 142666 last for a maximum of $\sim 2-3$ days (Zwintz et al. 2009). Comparing this to the orbital timescale at the inner disk rim (18.2 days), these scale height variations would be required to extend over a maximum of $\sim 10 \%-15 \%$ of the disk circumference. Furthermore, as the photometric variability is aperiodic, the scale height variations would have to rise and fall on timescales within the $\sim 18.2$ day orbital period. Taking this all into account, the $58^{\circ}$ disk inclination inferred for HD 142666 appears inconsistent with a disk-based origin for the UX Ori phenomena. In light of this, and the fact that the visibilities of the best-fit S:large TORUS model appear underresolved along baseline position angles that probe the disk minor axis, it seems likely that either the disk is inclined at $>58^{\circ}$ or that the UX Ori phenomena observed for HD 142666 is attributed to an outflow component of variable optical depth that is oriented perpendicular to the disk midplane. In both cases, additional NIRemitting material exterior to the flared disk we have considered here is required.

\section{Summary}

We have used geometric and radiative transfer modeling to explore the shape and structure of the inner rim of the disk of HD 142666. Our results are summarized as follows:

1. Fitting geometric models in which all of the circumstellar emission arises from the innermost regions of a disk to the $H$ - and $K$-band visibilities suggest a viewing geometry for HD 142666 of $i=58^{\circ}$ from face-on and major axis position angle of $160^{\circ}$ east of north. These values agree with previous interferometric modeling (Vural et al. 2014; Lazareff et al. 2017), VLT/NACO imaging (Garufi et al. 2017), and ALMA cycle 2 observations (Rubinstein et al. 2018) of the object. This viewing geometry was adopted in all our TORUS radiative transfer modeling.

2. The TORUS radiative transfer models we explore, which invoke vertical hydrostatic equilibrium in the circumstellar disk, are unable to reproduce the SED of HD 142666. This is consistent with previous results from radiation hydrodynamic and radiation hydrostatic modeling by Mulders \& Dominik (2012) and Flock et al. (2016) for other Herbig Ae/Be stars. Using a series of TORUS models without vertical hydrostatic equilibrium invoked, we further investigated whether the inflation of the inner disk to greater scale heights, induced by turbulence arising from MRI (Turner et al. 2014; Flock et al. 2017), for example, could reproduce the observed SED. Among the models we explored, we found that those in which small grains $(0.1 \mu \mathrm{m}$ in size $)$ are the largest grains in the inner disk rim and thus determine the rim location and shape are unable to simultaneously fit the NIR, MIR, and FIR portions of the SED. Instead, we found that our models required the presence of silicate dust of at least micrometer size to be present in the disk rim to be able to reproduce the SED across the full optical-to-millimeter wavelength range.

3. TORUS models invoking the existence of micron grains were also found to provide an improved fit to the NIR visibilities compared to the models including only small grains. This is consistent with the original study of the grain size dependence of the silicate dust destruction radius around Herbig Ae/Be stars by Monnier \& MillanGabet (2002). Furthermore, we found that models in which rim curvature arises due to the dependence of the dust sublimation temperature on the local gas density (Isella \& Natta 2005) provide improved fits to the visibilities $\left(\chi_{\mathrm{r}, \mathrm{vis}}^{2}=14.4\right)$ compared to those in which the rim curvature arises from grain-growth-induced settling (the THM07 models; Tannirkulam et al. 2007, $\chi_{\mathrm{r} \text {,vis }}^{2}=22.1$ ). In particular, the model providing the best fit to the SED and visibilities is the S:large model with disk scale height $h_{0 \text {,gas }}=7 \mathrm{au}$ and flaring parameter $\beta=1.09$. A slight improvement to the fit for the $S$ :large model is found using a viewing geometry of $i=58^{\circ}$ and $\mathrm{PA}_{\text {major }}=155^{\circ}\left(\chi_{\mathrm{r}}^{2}=13.94\right.$ assuming 337 degrees of freedom).

4. The visibility and closure phase residuals in the best-fit S:large TORUS model point to the presence of additional complexity to the NIR-emitting region which is unaccounted for in our TORUS models. The model closure phase signals are overestimated, indicating that the emission is more centrosymmetric while the model visibilities are underresolved along position angles tracing the apparent disk minor axis. In addition, we argue that the inclination we infer for HD 142666 is inconsistent with a disk-based origin for its UX Ori-type variability. This is further indication for the requirement of additional model complexity. Additional optically thick material present in dusty disk winds (Alexander \& Armitage 2007; Bans \& Königl 2012) and/or the gaseous disk material interior to the dust sublimation rim (Tannirkulam et al. 2008a, 2008b), for example, appear to be required.

C.L.D., S.K., A.K., and A.L. acknowledge support from the ERC Starting Grant "ImagePlanetFormDiscs" (grant agreement No. 639889), STFC Rutherford fellowship/grant (ST/ J004030/1, ST/K003445/1), and Philip Leverhulme Prize (PLP-2013-110). J.D.M., F.B., and B.K. acknowledge support from NSF grants AST-1210972 and AST-1506540. We would like to thank Bernard Lazareff, Jean-Baptiste Le Bouquin, and Rachel Akeson for their assistance in acquiring archival data 
for HD 142666. This work is based upon observations obtained with the Georgia State University Center for High Angular Resolution Astronomy Array at Mount Wilson Observatory. The CHARA Array is supported by the National Science Foundation under grant No. AST-1211929. Institutional support has been provided from the GSU College of Arts and Sciences and the GSU Office of the Vice President for Research and Economic Development. The calculations for this paper were performed on the University of Exeter Supercomputer, a DiRAC Facility jointly funded by STFC, the Large Facilities Capital Fund of BIS, and the University of Exeter. This research has made use of the NASA/IPAC Infrared Science Archive, which is operated by the Jet Propulsion Laboratory, California Institute of Technology, under contract with the National Aeronautics and Space Administration; the Keck Observatory Archive (KOA), which is operated by the W. M. Keck Observatory and the NASA Exoplanet Science Institute (NExScI), under contract with the National Aeronautics and Space Administration; the Jean-Marie Mariotti Center OiDBservice ${ }^{11}$; NASA's Astrophysics Data System; the VizieR catalog access tool, CDS, Strasbourg, France; the SIMBAD database, operated at CDS, Strasbourg, France; NumPy (Van Der Walt et al. 2011); matplotlib, a Python library for publication quality graphics (Hunter 2007); Astropy, a community-developed core Python package for Astronomy (Astropy Collaboration et al. 2013); the Jean-Marie Mariotti Center SearchCal service ${ }^{12}$ co-developed by LAGRANGE and IPAG; and the CDS Astronomical Databases SIMBAD and VIZIER. ${ }^{13}$ This work has made use of services produced by the NASA Exoplanet Science Institute at the California Institute of Technology.

Facilities: VLTI, CHARA, Keck: Interferometer.

Software: TORUS (Harries 2000; Harries et al. 2004; Kurosawa et al. 2006; Tannirkulam et al. 2007), pysynphot (STScI Development Team 2013), NumPy (Van Der Walt et al. 2011), matplotlib (Hunter 2007), Astropy (Astropy Collaboration et al. 2013).

\section{Appendix Multiband Photometry}

The photometries used to build the SED for HD 142666 are listed in Table 9. These have been flux-converted, where necessary, using central wavelengths and zero-point magnitudes from Mann \& von Braun (2015) and Cutri et al. (2003, 2012a).

\section{ORCID iDs}

Claire L. Davies (iD https://orcid.org/0000-0001-9764-2357

Stefan Kraus (iD https://orcid.org/0000-0001-6017-8773

Tim J. Harries (iD https://orcid.org/0000-0001-8228-9503

Alexander Kreplin (iD https://orcid.org/0000-0002-0911-9505

John D. Monnier (ib https://orcid.org/0000-0002-3380-3307

Brian Kloppenborg (iD https://orcid.org/0000-0003-0350-5453

David M. Acreman (1) https://orcid.org/0000-0002-4881-7584

Fabien Baron (ib https://orcid.org/0000-0002-5074-1128

Rafael Millan-Gabet (D) https://orcid.org/0000-0003-

0447-5866

\footnotetext{
${ }_{11}$ Available at http://oidb.jmmc.fr.

12 Available at http://www.jmmc.fr/searchcal.

13 Available at http://cdsweb.u-strasbg.fr/.
}

Table 9

Photometry for HD 142666

\begin{tabular}{|c|c|c|}
\hline $\begin{array}{l}\lambda \\
(\mu \mathrm{m})\end{array}$ & $\begin{array}{c}\text { Flux } \\
(\mathrm{Jy})\end{array}$ & Reference \\
\hline 0.422 & $0.65 \pm 0.012$ & Høg et al. (2000) \\
\hline 0.44 & $0.53 \pm 0.024$ & Tannirkulam et al. (2008a) \\
\hline 0.535 & $1.07 \pm 0.02$ & Høg et al. (2000) \\
\hline 0.55 & $0.86 \pm 0.03$ & Tannirkulam et al. (2008a) \\
\hline 0.71 & $0.96 \pm 0.05$ & Tannirkulam et al. (2008a) \\
\hline 0.7625 & $1.46 \pm 1.34$ & Zacharias et al. (2013) \\
\hline 0.79 & $1.14 \pm 0.04$ & Tannirkulam et al. (2008a) \\
\hline 1.235 & $1.83 \pm 0.04$ & Ita et al. (2010) \\
\hline 1.662 & $2.06 \pm 0.05$ & Ita et al. (2010) \\
\hline 2.159 & $2.47 \pm 0.04$ & Ita et al. (2010) \\
\hline 3.368 & $3.04 \pm 0.20$ & Cutri et al. (2012b) \\
\hline 4.618 & $3.78 \pm 0.17$ & Cutri et al. (2012b) \\
\hline 9.0 & $5.15 \pm 0.36$ & Ishihara et al. (2010) \\
\hline 12.0 & $8.57 \pm 4.00$ & Helou \& Walker (1988) \\
\hline 12.082 & $7.20 \pm 0.07$ & Cutri et al. (2012b) \\
\hline 18.0 & $6.58 \pm 0.01$ & Ishihara et al. (2010) \\
\hline 25.0 & $11.20 \pm 6.00$ & Helou \& Walker (1988) \\
\hline 60.0 & $7.47 \pm 5.00$ & Moshir et al. (1990) \\
\hline 65.0 & $5.26 \pm 0.37$ & Yamamura et al. (2010) \\
\hline 70.0 & $6.56 \pm 0.33$ & Pascual et al. (2016) \\
\hline 90.0 & $5.73 \pm 0.32$ & Yamamura et al. (2010) \\
\hline 100.0 & $5.91 \pm 0.30$ & Pascual et al. (2016) \\
\hline 140.0 & $5.97 \pm 0.83$ & Yamamura et al. (2010) \\
\hline 160.0 & $4.33 \pm 0.22$ & Pascual et al. (2016) \\
\hline 450.0 & $1.09 \pm 0.06$ & Sylvester et al. (1996) \\
\hline 800.0 & $0.35 \pm 0.02$ & Sylvester et al. (1996) \\
\hline 850.0 & $0.26 \pm 0.08$ & Di Francesco et al. (2008) \\
\hline 1100.0 & $0.18 \pm 0.01$ & Sylvester et al. (1996) \\
\hline 1200.0 & $0.079 \pm 0.004$ & Natta et al. (2004) \\
\hline 1300.0 & $0.127 \pm 0.009$ & Sylvester et al. (1996) \\
\hline 3100.0 & $0.013 \pm 0.001$ & Natta et al. (2004) \\
\hline 3300.0 & $0.011 \pm 0.001$ & Natta et al. (2004) \\
\hline
\end{tabular}

\section{References}

Akeson, R. L., Ciardi, D. R., van Belle, G. T., Creech-Eakman, M. J., \& Lada, E. A. 2000, ApJ, 543, 313

Alexander, R. D., \& Armitage, P. J. 2007, MNRAS, 375, 500

Andrews, S. M., Rosenfeld, K. A., Kraus, A. L., \& Wilner, D. J. 2013, ApJ, 771,129

Astropy Collaboration, Robitaille, T. P., Tollerud, E. J., et al. 2013, A\&A, 558, A33

Bailer-Jones, C. A. L., Rybizki, J., Fouesneau, M., Mantelet, G., \& Andrae, R. 2018, AJ, 156, 58

Bans, A., \& Königl, A. 2012, ApJ, 758, 100

Beckwith, S. V. W., Sargent, A. I., Chini, R. S., \& Guesten, R. 1990, AJ, 99, 924

Bonneau, D., Clausse, J.-M., Delfosse, X., et al. 2006, A\&A, 456, 789

Bonneau, D., Delfosse, X., Mourard, D., et al. 2011, A\&A, 535, A53

Cardelli, J. A., Clayton, G. C., \& Mathis, J. S. 1989, ApJ, 345, 245

Colavita, M. M., Wizinowich, P. L., Akeson, R. L., et al. 2013, PASP, 125,1226

Cutri, R. M., Skrutskie, M. F., van Dyk, S., et al. 2003, yCat, 2246

Cutri, R. M., Wright, E. L., Conrow, T., et al. 2012a, Explanatory Supplement to the WISE All-Sky Data Release Products, Tech. Rep., http://adsabs. harvard.edu/abs/2012wise.rept....1C

Cutri, R. M., Wright, E. L., Conrow, T., et al. 2012b, yCat, 2311

Dent, W. R. F., Greaves, J. S., \& Coulson, I. M. 2005, MNRAS, 359, 663

Di Francesco, J., Johnstone, D., Kirk, H., MacKenzie, T., \& Ledwosinska, E. 2008, ApJS, 175, 277

Dominik, C., Dullemond, C. P., Waters, L. B. F. M., \& Walch, S. 2003, A\&A, 398, 607

Draine, B. T. 2003, ApJ, 598, 1026

Draine, B. T., \& Lee, H. M. 1984, ApJ, 285, 89

Dullemond, C. P., Dominik, C., \& Natta, A. 2001, ApJ, 560, 957 
Duvert, G., Young, J., \& Hummel, C. A. 2017, A\&A, 597, A8

Eisner, J. A., Graham, J. R., Akeson, R. L., \& Najita, J. 2009, ApJ, 692, 309 Fischer, W., Edwards, S., Hillenbrand, L., \& Kwan, J. 2011, ApJ, 730, 73 Flock, M., Fromang, S., Turner, N. J., \& Benisty, M. 2016, ApJ, 827, 144 Flock, M., Fromang, S., Turner, N. J., \& Benisty, M. 2017, ApJ, 835, 230 Gaia Collaboration, Brown, A. G. A., Vallenari, A., et al. 2018, A\&A, 616, A1 Gaia Collaboration, Prusti, T., de Bruijne, J. H. J., et al. 2016, A\&A, 595, A1 Garufi, A., Meeus, G., Benisty, M., et al. 2017, A\&A, 603, A21

Grinin, V. P., Kiselev, N. N., Minikulov, N. K., Chernova, G. P., \& Voshchinnikov, N. V. 1991, Ap\&SS, 186, 283

Guimarães, M. M., Alencar, S. H. P., Corradi, W. J. B., \& Vieira, S. L. A. 2006, A\&A, 457, 581

Harries, T. J. 2000, MNRAS, 315, 722

Harries, T. J., Monnier, J. D., Symington, N. H., \& Kurosawa, R. 2004, MNRAS, 350, 565

Haubois, X., Bernaud, P., Mella, G., et al. 2014, Proc. SPIE, 9146, 91460 O

Helou, G., \& Walker, D. W. (ed.) 1988, Infrared Astronomical Satellite (IRAS) Catalogs and Atlases. Volume 7: The Small Scale Structure Catalog, Vol. 7, NASA RP-1190 (Washington, DC: NASA)

Herbig, G. H. 1960, ApJS, 4, 337

Herbst, W., \& Shevchenko, V. S. 1999, AJ, 118, 1043

Hernández, J., Calvet, N., Briceño, C., Hartmann, L., \& Berlind, P. 2004, AJ, 127,1682

Hildebrand, R. H. 1983, QJRAS, 24, 267

Hillenbrand, L. A., Strom, S. E., Vrba, F. J., \& Keene, J. 1992, ApJ, 397, 613

Høg, E., Fabricius, C., Makarov, V. V., et al. 2000, A\&A, 355, L27

Houck, J. R., Roellig, T. L., van Cleve, J., et al. 2004, ApJS, 154, 18

Hunter, J. D. 2007, CSE, 9, 90

Ilee, J. D., Cyganowski, C. J., Nazari, P., et al. 2016, MNRAS, 462, 4386

Ilee, J. D., Fairlamb, J., Oudmaijer, R. D., et al. 2014, MNRAS, 445, 3723

Isella, A., \& Natta, A. 2005, A\&A, 438, 899

Ishihara, D., Onaka, T., Kataza, H., et al. 2010, A\&A, 514, A1

Ita, Y., Matsuura, M., Ishihara, D., et al. 2010, A\&A, 514, A2

Kama, M., Min, M., \& Dominik, C. 2009, A\&A, 506, 1199

Kamp, I., \& Dullemond, C. P. 2004, ApJ, 615, 991

Keller, L. D., Sloan, G. C., Forrest, W. J., et al. 2008, ApJ, 684, 411

Kim, S.-H., Martin, P. G., \& Hendry, P. D. 1994, ApJ, 422, 164

Kraus, S., Hofmann, K.-H., Malbet, F., et al. 2009, A\&A, 508, 787

Kraus, S., Kluska, J., Kreplin, A., et al. 2017, ApJL, 835, L5

Kraus, S., Monnier, J., Harries, T., et al. 2014, Proc. SPIE, 9146, 914611

Kraus, S., Preibisch, T., \& Ohnaka, K. 2008, ApJ, 676, 490

Kreplin, A., Madlener, D., Chen, L., et al. 2016, A\&A, 590, A96

Kreplin, A., Weigelt, G., Kraus, S., et al. 2013, A\&A, 551, A21

Kurosawa, R., Harries, T. J., \& Symington, N. H. 2006, MNRAS, 370, 580

Kurucz, R. L. 1979, ApJS, 40, 1

Lazareff, B., Berger, J.-P., Kluska, J., et al. 2017, A\&A, 599, A85

Le Bouquin, J.-B., Berger, J.-P., Lazareff, B., et al. 2011, A\&A, 535, A67

Lindegren, L., Lammers, U., Bastian, U., et al. 2016, A\&A, 595, A4

Lucy, L. B. 1999, A\&A, 344, 282

Makarov, V., Bastian, U., Hoeg, E., Grossmann, V., \& Wicenec, A. 1994, IBVS, 4118, 1

Mamajek, E. E. 2012, ApJL, 754, L20

Mann, A. W., \& von Braun, K. 2015, PASP, 127, 102

Manoj, P., Bhatt, H. C., Maheswar, G., \& Muneer, S. 2006, ApJ, 653, 657

McClure, M. K., D’Alessio, P., Calvet, N., et al. 2013, ApJ, 775, 114

McDonald, I., Zijlstra, A. A., \& Watson, R. A. 2017, MNRAS, 471, 770

Meeus, G., Waelkens, C., \& Malfait, K. 1998, A\&A, 329, 131

Meeus, G., Waters, L. B. F. M., Bouwman, J., et al. 2001, A\&A, 365, 476
Millan-Gabet, R., Schloerb, F. P., Traub, W. A., et al. 1999, ApJL, 513, L131

Monnier, J. D., Berger, J.-P., Millan-Gabet, R., et al. 2006, ApJ, 647, 444

Monnier, J. D., \& Millan-Gabet, R. 2002, ApJ, 579, 694

Monnier, J. D., Millan-Gabet, R., Billmeier, R., et al. 2005, ApJ, 624, 832

Mora, A., Merín, B., Solano, E., et al. 2001, A\&A, 378, 116

Moshir, M., Copan, G., Conrow, T., et al. 1990, IRAS Faint Source Catalogue, Version 2.0

Mulders, G. D., \& Dominik, C. 2012, A\&A, 539, A9

Natta, A., Grinin, V. P., Mannings, V., \& Ungerechts, H. 1997, ApJ, 491, 885

Natta, A., Prusti, T., Neri, R., et al. 2001, A\&A, 371, 186

Natta, A., Testi, L., Neri, R., Shepherd, D. S., \& Wilner, D. J. 2004, A\&A, 416, 179

Newville, M., Stensitzki, T., Allen, D. B., \& Ingargiola, A. 2014, LMFIT: NonLinear Least-Square Minimization and Curve-Fitting for Python, Zenodo, doi:10.5281/zenodo.11813

Pascual, N., Montesinos, B., Meeus, G., et al. 2016, A\&A, 586, A6

Pauls, T. A., Young, J. S., Cotton, W. D., \& Monnier, J. D. 2005, PASP, 117,1255

Pecaut, M. J., \& Mamajek, E. E. 2013, ApJS, 208, 9

Pollack, J. B., Hollenbach, D., Beckwith, S., et al. 1994, ApJ, 421, 615

Pontoppidan, K. M., Dullemond, C. P., Blake, G. A., et al. 2007, ApJ, 656, 980

Ricci, L., Testi, L., Natta, A., et al. 2014, ApJ, 791, 20

Rubinstein, A. E., Macias, E., Espaillat, C. C., et al. 2018, ApJ, 860, 7

Schegerer, A. A., Ratzka, T., Schuller, P. A., et al. 2013, A\&A, 555, A103

Shakura, N. I., \& Sunyaev, R. A. 1973, A\&A, 24, 337

Siess, L., Dufour, E., \& Forestini, M. 2000, A\&A, 358, 593

Strom, S. E., Strom, K. M., Yost, J., Carrasco, L., \& Grasdalen, G. 1972, ApJ, 173,353

STScI Development Team 2013, pysynphot: Synthetic photometry software package, Astrophysics Source Code Library, ascl:1303.023

Sylvester, R. J., Skinner, C. J., Barlow, M. J., \& Mannings, V. 1996, MNRAS, 279,915

Tambovtseva, L. V., \& Grinin, V. P. 2008, AstL, 34, 231

Tannirkulam, A., Harries, T. J., \& Monnier, J. D. 2007, ApJ, 661, 374

Tannirkulam, A., Monnier, J. D., Harries, T. J., et al. 2008a, ApJ, 689, 513

Tannirkulam, A., Monnier, J. D., Millan-Gabet, R., et al. 2008b, ApJL, 677, L51

ten Brummelaar, T. A., McAlister, H. A., Ridgway, S. T., et al. 2005, ApJ, 628,453

ten Brummelaar, T. A., Sturmann, J., McAlister, H. A., et al. 2012, Proc. SPIE, $8445,84453 \mathrm{C}$

ten Brummelaar, T. A., Sturmann, J., Ridgway, S. T., et al. 2013, JAI, 2 , 1340004

Testi, L., Birnstiel, T., Ricci, L., et al. 2014, in Protostars and Planets VI, ed. H. Beuther et al. (Tucson, AZ: Univ. Arizona Press), 339

Turner, N. J., Benisty, M., Dullemond, C. P., \& Hirose, S. 2014, ApJ, 780, 42 van Boekel, R., Waters, L. B. F. M., Dominik, C., et al. 2003, A\&A, 400, L21 Van Der Walt, S., Colbert, S. C., \& Varoquaux, G. 2011, CSE, 13, 22 Vinković, D., \& Jurkić, T. 2007, ApJ, 658, 462

Vural, J., Kreplin, A., Kishimoto, M., et al. 2014, A\&A, 564, A118

Walker, C., Wood, K., Lada, C. J., et al. 2004, MNRAS, 351, 607

Walker, H. J., \& Wolstencroft, R. D. 1988, PASP, 100, 1509

Whitney, B. A., Indebetouw, R., Bjorkman, J. E., \& Wood, K. 2004, ApJ, 617,1177

Wood, K., Wolff, M. J., Bjorkman, J. E., \& Whitney, B. 2002, ApJ, 564, 887 Yamamura, I., Makiuti, S., Ikeda, N., et al. 2010, yCat, 2298

Zacharias, N., Finch, C. T., Girard, T. M., et al. 2013, AJ, 145, 44

Zwintz, K., Kallinger, T., Guenther, D. B., et al. 2009, A\&A, 494, 1031 